\title{
THE LOCAL STRUCTURE OF POISSON MANIFOLDS
}

\author{
ALAN WEINSTEIN
}

Table of Contents

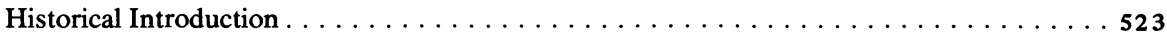

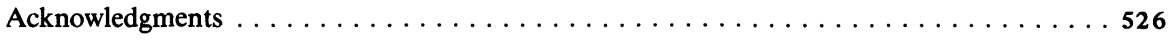

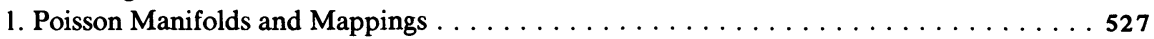

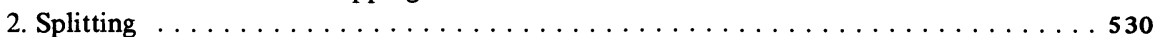

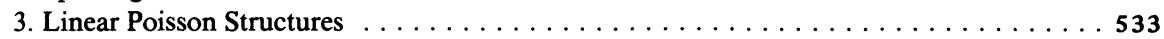

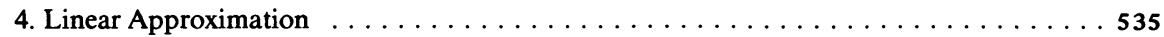

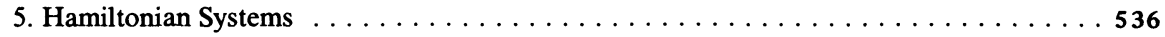

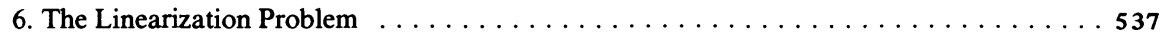

7. Function Groups, Realizations, and Momentum Mappings . . . . . . . . . . . 540

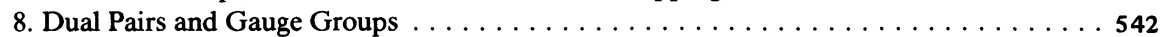

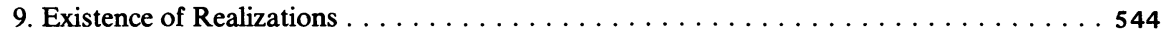

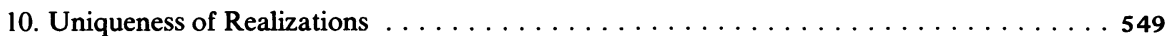

11. The Restricted Three Body Problems and Other Examples . . . . . . . . . 550

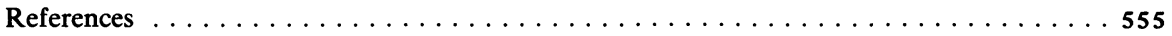

\section{Historical Introduction}

The classical Poisson bracket operation defined on functions on $\mathbf{R}^{2 n}$ is

$$
\{f, g\}=\sum_{i, j=1}^{n}\left(\frac{\partial f}{\partial q_{i}} \frac{\partial g}{\partial p_{i}}-\frac{\partial g}{\partial q_{i}} \frac{\partial f}{\partial p_{i}}\right) .
$$

In the early nineteenth century, Poisson noticed that the vanishing of $\{f, g\}$ and $\{f, h\}$ imply that of $\{f,\{g, h\}\}$; almost thirty years later Jacobi discovered the identity $\{f,\{g, h\}\}=\{\{f, g\}, h\}+\{g,\{f, h\}\}$ which "explains" Poisson's theorem. In his study of general composition laws satisfying the Jacobi identity, Lie [29] defined in local coordinate form what is now known as a Poisson structure. On $\mathbf{R}^{r}$ such a structure is given by functions $w_{i j}\left(x_{1}, \cdots, x_{r}\right)$ satisfying the identities

$$
\begin{gathered}
w_{i j}+w_{j i}=0 \\
\sum_{l=1}^{r}\left(w_{l j} \frac{\partial w_{i k}}{\partial x_{l}}+w_{l i} \frac{\partial w_{k j}}{\partial x_{l}}+w_{l k} \frac{\partial w_{j i}}{\partial x_{l}}\right)=0
\end{gathered}
$$

Received September 17, 1982. Research supported by the Miller Institute and National Science Foundation Grant MCS 80-23356. 
which imply that the bilinear operation

$$
\{F, G\}=\sum_{i, j=1}^{r} w_{i j} \frac{\partial F}{\partial x_{i}} \frac{\partial G}{\partial x_{j}}
$$

is antisymmetric and satisfies the Jacobi identity; i.e., the algebra of functions $C^{\infty}\left(\mathbf{R}^{r}\right)$ becomes a Lie algebra. An abstractly defined Lie algebra structure $\{$, on $C^{\infty}\left(\mathbf{R}^{r}\right)$ arises in this way if and only if it satisfies the Leibniz identity $\{F, G H\}=\{F, G\} H+G\{F, H\}$, and this enables us to define a Poisson structure on a manifold $P$ to be a Lie algebra structure $\{$,$\} on C^{\infty}(P)$ which satisfies the Leibniz identity. The functions $w_{i j}$ may then be seen as the components in local coordinates of an antisymmetric contravariant 2-tensor $w$; the Jacobi identity may be interpreted as the vanishing on $w$ of a certain natural quadratic differential operator of first order. (Berezin [5], Hermann [19], Lichnerowicz [28], Tulczejew [44]).

Poisson structures have recently become interesting in connection with completely integrable systems and for the hamiltonian formulation of field theoreis in physical variables. (See the papers in Tabor and Treve [43] and references therein.) The aim of this paper is to develop the theory of Poisson manifolds with an eye toward these applications and also a new applicationthe study of singular limits of hamiltonian systems.

The natural setting for hamiltonian systems is on symplectic manifolds. These may be described as manifolds carrying a Poisson structure which is locally isomorphic to the standard one on an $\mathbf{R}^{2 n}$; the coordinates $\left(q_{1}, \cdots, q_{n}, p_{1}, \cdots, p_{n}\right)$ are then called canonical variables. (There is an extension of this definition to infinite dimensions, which we ignore for now.) A criterion for a Poisson structure to be symplectic is that the tensor $w$ (or the matrix $\left(w_{i j}\right)$ ) have rank everywhere equal to the dimension of the manifold.

The simplest example of a nonsymplectic Poisson structure is $\mathbf{R}^{2 n+s}$ with variables $\left(q_{1}, \cdots, q_{n}, p_{1}, \cdots, p_{n}, c_{1}, \cdots, c_{s}\right)$ and Poisson brackets given by the standard formula (*). Functions of $\left(c_{1}, \cdots, c_{s}\right)$ have zero Poisson bracket with everything and are called by Lie distinguished functions. (Nowadays they are called invariants or Casimir functions.) Each manifold on which all $c_{j}$ are constant inherits a Poisson structure which is then nondegenerate, so $\mathbf{R}^{2 n+s}$ may be thought of as foliated by symplectic manifolds. This turns out to be the situation at the generic points of any Poisson manifold: Lie proved that any Poisson structure on $\mathbf{R}^{2 n+s}$ for which the rank of $w_{i j}$ is constant ${ }^{1}$ and equal to $2 n$ admits $s$ independent distinguished functions and so is locally isomorphic

\footnotetext{
'Actually, Lie assumed the constancy of rank without saying it explicitly.
} 
to the standard example just described; furthermore, the rank of any Poìsson structure is constant (and even) on the open subset where it attains its maximal value. $^{2}$

Another important example of a Poisson structure, also introduced by Lie, arises when we are given the structure constants of a Lie algebra, i.e., $r^{3}$ constants $c_{i j k}$ satisfying the identities

$$
c_{i j k}+c_{j i k}=0, \quad c_{i j j}+c_{j k i}+c_{k i j}=0 .
$$

The functions $w_{i j}(x)=\sum_{k=1}^{r} c_{i j k} x_{k}$ then define a Poisson structure on $\mathbf{R}^{r}{ }^{3}$ The rank of $\left(w_{i j}\right)$ is no longer constant (e.g. it is zero at $\left.x=0\right)$, so Lie's theory applies only at the "regular points" where the rank is maximal. This was enough for Lie to prove his "third theorem" on the existence of a local group with given structure constants and to classify locally the $r$ 'tuples $\left(\phi_{1}, \cdots, \phi_{r}\right)$ of functions on $\mathbf{R}^{2 n}$ satisfying the bracket relations $\left\{\phi_{i}, \phi_{j}\right\}=\sum_{k=1}^{r} c_{i j k} \phi_{k}$. In the course of this classification, Lie observed that the leaves of the symplectic foliation of $\mathbf{R}^{r}$ were just the "smallest invariant submanifolds for the dual of the adjoint group" acting on $\mathbf{R}^{r}$.

Nowadays we think of $\mathbf{R}^{r}$ as the dual space $g^{*}$ of the Lie algebra $(g,[]$, whose basis $X_{1}, \cdots, X_{k}$ satisfies $\left[X_{i}, X_{j}\right]=\Sigma_{k=1}^{r} c_{i j k} X_{k}$. The "dual of the adjoint group" is called the coadjoint representation, and the smallest invariant manifolds are the coadjoint orbits. ${ }^{4}$ Their symplectic structure was rediscovered in the 1960's by Kirillov [24], Kostant [26], and Souriau [41], whose arguments covered the singular coadjoint orbits as well as the regular ones. In fact it turns out (see Kirillov [25]) that through each point of every Poisson manifold there passes a symplectic manifold whose dimension equals the rank of the Poisson structure there, and the Poisson structure is built up out of the Poisson structures on these symplectic leaves. For a $g^{*}$ carrying Lie's Poisson structure, the symplectic leaves are just the coadjoint orbits.

This property of being smoothly decomposed into symplectic manifolds of different dimensions seems to make Poisson manifolds an appropriate setting for studying a phenomenon which is quite common in mechanics: if a mechanical system is modeled by a symplectic manifold, then when a parameter in the system reaches a limiting value (usually 0 or $\infty$ ), the limiting system also has a symplectic formulation, but with fewer degrees of freedom. Examples of this are:

(i) the restricted 3-body problem in celestial mechanics (mass $\rightarrow 0$ );

\footnotetext{
${ }^{2}$ These results were rediscovered by Lichnerowicz [28] and Hermann [20].

${ }^{3}$ Introduced again by Berezin [4].

${ }^{4}$ By the way, Lie also discovered and used, more or less as it is used now, the momentum mapping and its equivariance under the coadjoint representation.
} 
(ii) the guiding center limit for a particle in an electromagnetic field (charge/mass $\rightarrow \infty$ );

(iii) the limit of discrete vortices in the motion of incompressible fluids (concentration of vorticity $\rightarrow \infty$ );

(iv) the classical limit of quantum mechanics $(\hbar \rightarrow 0)$.

There are also examples when the number of degrees of freedom remains the same but the global structure of a symplectic manifold of group changes, as in the newtonian limit of special relativity $(c \rightarrow \infty)$; these too should be accessible to study in terms of Poisson structures.

A good part of this paper then will consist of an extension of Lie's results to the case of variable rank. Most of the methods are ones which Lie himself would have used, but the geometric language developed since his time gives us new insight and enables us to say some things more efficiently.

There is one new aspect to the theory which is peculiar to the case of variable rank. At a point where the functions $w_{i j}(x)$ vanish, we can linearize them to obtain a linear Poisson structure living on the tangent space. The linearization question is whether the original Poisson structure is locally equivalent to a linear one. The question has different answers according to whether we work in the formal, analytic, or $C^{\infty}$ categories, and there remain some unsolved problems.

Some interesting work on the singular structure of Poisson manifolds, from a more algebraic viewpoint than ours, can be found in the papers of Vinogradov and Krasilshchik [46] and Berger [6].

To end this introduction, the author wishes to point out that a great deal of interest today in symplectic actions and coadjoint orbits lies in their relevance for understanding unitary actions of Lie groups. This is truly a modern development for which there does not seem to be any precedent in Lie's work.

\section{Acknowledgments}

Bob Hermann and Wilfrid Schmid, in conversations and their published work (Hermann [20], Schmid [40]) pointed the author toward Lie's [29] pioneering work on Poisson manifolds. Hans Duistermaat was responsible for my recent education in the geometry of Lie groups, some of which found direct applications in this paper. The author's interest in applications of Poisson manifolds to the understanding of variational principles and Clebsch variables was stimulated at a conference at the La Jolla Institute (December 1981) organized by Michael Tabor and Yvain Treve. Conversations there with Frank 
Henyey, Darryl Holm, John Hubbard, Boris Kupershmidt, and Robert Littlejohn were especially helpful. Finally, Jerry Marsden has been a patient listener and frequent collaborator in the work described here-our joint work described in Marsden and Weinstein [32] and Marsden, Ratiu and Weinstein [33] is a close companion to the present paper.

\section{Poisson manifolds and mappings}

A Poisson structure on a manifold $P$ is defined as a Lie algebra structure $\{$, $\}$ on $C^{\infty}(P)$ satisfying the Liebniz identity $\{F G, H\}=F\{G, H\}+\{F, H\} G$. The bracket operation $\{$,$\} is thus a derivation in each entry, and so in$ particular for each function $H$ there is a vector field $\xi_{H}$ such that $\xi_{H} \cdot F=$ $\{F, H\}$ for all $F . \xi_{H}$ is called the hamiltonian vector field generated by $H$. At any point $p \in P$, the value of $\{F, H\}$ and hence of $\xi_{H}$ depends only on the differential of $H$, so there is a bundle map $B: T^{*} P \rightarrow T P$ such that $\xi_{H}=B \circ d H$ for all $H$. We may also think of $B$ as defining a contravariant antisymmetric 2-tensor $w$ in $P$, for which $\{F, G\}=\langle(d F, d G), w\rangle$. The tensor $w$ is sometimes called a cosymplectic structure; the Jacobi identity for $\{$,$\} is equivalent to the$ vanishing of the so-called Schouten [42] bracket [ $w, w]$.

In local coordinates $\left(x_{1}, \cdots, x_{r}\right)$, a Poisson structure is determined by the component functions $w_{i j}(x)$ of $w$. In terms of the bracket we have simply $\left\{x_{i}, x_{j}\right\}=w_{i j}(x)$; in other words, the Poisson structure is specified if we give the bracket relations satisfied by the coordinate functions. This is exactly how Lie thought of Poisson structures; he noted that the bracket

$$
\{F, G\}=\sum_{i, j=1}^{r} w_{i j} \frac{\partial F}{\partial x_{i}} \frac{\partial G}{\partial x_{j}}
$$

satisfies the asymmetry and Jacobi conditions if and only if $w_{i j}=-w_{j i}$ and

$$
\sum_{l=1}^{r}\left(w_{l j} \frac{\partial w_{i k}}{\partial x_{l}}+w_{l i} \frac{\partial w_{k j}}{\partial x_{l}}+w_{l k} \frac{\partial w_{j l}}{\partial x_{l}}\right)=0 .
$$

It follows from the Jacobi identity that the (local) flow of each $\xi_{H}$ preserves the Poisson structure, and also that $\xi_{\{H, K\}}=\xi_{K} \xi_{H}-\xi_{H} \xi_{K}=\left[\xi_{H}, \xi_{K}\right]$; we use Arnold's [3] sign convention for the bracket of vector fields. Thus the Poisson structure determines a Lie algebra homomorphism from $C^{\infty}(P)$ to the infinitesimal automorphisms of the Poisson structure.

Automorphisms of a Poisson manifold are an example of Poisson mappings defined in general as maps $J: P_{1} \rightarrow P_{2}$ between Poisson manifolds such that $\{F \circ J, G \circ J\}_{1}=\{F, G\}_{2} \circ J$, or equivalently by the condition $J_{*} w_{1}(x)=$ $w_{2}(J(x))$. A Poisson submanfold is a submanifold $Q$ in a Poisson manifold $P$ 
with a Poisson structure for which the inclusion is a Poisson mapping. Such a structure, if it exists, is unique.

Lemma 1.1. $Q \subseteq P$ is a Poisson submanifold if and only if each tangent space $T_{x} Q$ contains the image of $B_{x}: T_{x}^{*} P \rightarrow T_{x} P$, i.e., if and only if all hamiltonian vector fields are tangent to $Q$.

Proof. Given functions $\bar{F}$ and $\bar{G}$ on $Q$, extend them to functions $F$ and $G$ on $P$. (It will be enough to do this locally.) The tangency condition implies that the restriction of $\{F, G\}$ to $Q$ depends only on $\bar{F}$ and $\bar{G}$, so there is an induced bracket operation on $Q$ which is easily seen to make $Q$ into a Poisson submanifold. If the tangency condition fails, the bracket of extended functions depends upon the extensions, so the restriction map $C^{\infty}(P) \rightarrow C^{\infty}(Q)$ cannot define a homomorphism for any Lie algebra structure on $C^{\infty}(Q)$.

A related fact is

Lemma 1.2. Let $J: P_{1} \rightarrow P_{2}$ be a Poisson mapping, and $H$ a function on $P_{2}$. Then the trajectories on $P$ of the hamiltonian vector field $\xi_{H}$ are the projections under $J$ of the trajectories on $P_{2}$ of $\xi_{H} \circ J$.

Proof. Let $\sigma(t)$ be an integral curve of $\xi_{H} \circ J$. Then for any function $G$ on $P_{2}$,

$$
\begin{aligned}
\frac{d}{d t}(G \circ(J \circ \sigma)) & =\frac{d}{d t}((G \circ J) \circ \sigma)=\{G \circ J, H \circ J\} \circ \sigma \\
& =(\{G, H\} \circ J) \circ \sigma=\{G, H\} \cdot(J \circ \sigma) .
\end{aligned}
$$

It follows that $J \circ \sigma$ is an integral curve of $\xi_{H}$. Clearly, all integral curves of $\xi_{H}$ arise in this way. q.e.d.

See Guillemin and Sternberg [15] and Mishchenko and Fomenko [34] for applications of this lemma to the study of collective and invariant motion.

The rank of a Poisson structure at a point $x \in P$ is defined to be the rank of $B_{x}: T_{x}^{*} P \rightarrow T_{x} P$. (In local coordinates it is the rank of the matrix $w_{i j}(x)$.) The invariance of the Poisson structure under hamiltonian flows implies the constancy of rank along the orbits of such flows. From this it is not hard to derive the following result, due to Kirillov [25] (see also Hermann [17]) in general but to Lie [29] for the case of constant rank: Every Poisson manifold is essentially a union of symplectic manifolds which fit together in a smooth way.

Proposition 1.3. Define a relation $\sim$ on $P$ by declaring $x \sim y$ if $y$ can be reached from $x$ by a piecewise smooth curve, each segment of which is a trajectory of a hamiltonian vector field. Then $\sim$ is an equivalence relation, and the equivalence classes are Poisson submanifolds of $P$. The bracket of functions on $P$ is therefore determined by the brackets of their restrictions to these submanifolds. The dimension of each such submanifold $Q$ is equal to the rank of the Poisson structure (of $P$ or of $Q$ ) at each point of $Q$. 
A Poisson structure for which the rank is everywhere equal to the dimension of the manifold is called nondegenerate or symplectic. The symplectic structure on such a manifold is the 2-form $\Omega$ defined by $\Omega(\xi, \eta)=w\left(B^{-1} \xi, B^{-1} \eta\right)$; the usual Poisson brackets and hamiltonian vector fields for this symplectic structure are just the ones for the Poisson structure. We shall call the equivalence classes $Q$ in Proposition 1.3 the symplectic leaves of $P$, since they form a foliation everywhere that the rank of the Poisson structure is locally constant (for instance on the open dense subset of points with locally maximal rank).

A Casimir function, or invariant, on a Poisson manifold is a function $C$ such that $\{C, F\}=0$ for all functions $F$; equivalent conditions are that $C$ is constant along the orbits of all hamiltonian vector fields or that the hamiltonian vector field of $C$ itself is zero. A Casimir function is constant along each symplectic leaf, and in a region where the rank is constant, the symplectic leaves are exactly the common level manifolds of the local Casimir functions.

It is sometimes possible to define an induced Poisson structure on a submanifold which is not a Poisson submanifold. For instance, only Poisson submanifolds of a symplectic manifold are its connected components, but any submanifold on which the symplectic form is nondegenerate has an induced symplectic, hence Poisson structure. The following proposition will be useful in the next section.

Proposition 1.4. Let $Q$ be a submanifold of the Poisson manifold $P$ such that the following conditions are satisfied at each $x \in Q$ :

(i) $B_{x}\left(T_{x} Q^{\perp}\right) \cap T_{x} Q=\{0\}$,

(ii) $T_{x} Q^{\perp} \cap \operatorname{Ker} B_{x}=\{0\}$,

where $T_{x} Q^{\perp}$ is the annihilator of $T_{x} Q$ in $T_{x}^{*} P$. Then there is a naturally induced Poisson structure on $Q$.

Proof. Condition (ii) is equivalent to $T_{x} Q+\operatorname{Im} B_{x}=T_{x} P$, i.e., $Q$ intersects each symplectic leaf transversely. Thus $Q$ is a union of manifolds, each of which is a submanifold of a symplectic manifold. Now the intersection $B_{x}\left(T_{x} Q^{\perp}\right) \cap T_{x} Q$ is just the null space of the induced symplectic form on Im $B_{x} \cap T_{x} Q$, so condition (i) tells us that the intersection manifolds making up $Q$ are in fact symplectic. Thus we can put together the Poisson brackets on these manifolds to get a bracket on $Q$. To see that the resulting structure is smooth, we note that conditions (i) and (ii) imply that $B_{x}\left(T_{x} Q^{\perp}\right) \oplus T_{x} Q=T_{x} P$ for each $Q$, giving a smooth bundle projection $\pi$ from the restricted tangent bundle $T_{Q} P$ onto $T Q$. The induced Poisson structure on $Q$ is then defined by 
the composed map $T^{*} Q \stackrel{\pi^{*}}{\rightarrow} T_{Q}^{*} P \stackrel{B_{Q}}{\rightarrow} T_{Q} P \stackrel{\pi}{\rightarrow} T Q$. (The argument involving submanifolds was needed to show that the induced structure satisfies the Jacobi and Leibniz identities.)

\section{Splitting}

The product $P_{1} \times P_{2}$ becomes a Poisson manifold in an obvious way so that the projections $\pi_{j}: P_{1} \times P_{2} \rightarrow P_{j}$ are Poisson mappings, and $\pi_{1}\left(C^{\infty}\left(P_{1}\right)\right)$ and $\pi_{2}\left(C^{\infty}\left(P_{2}\right)\right)$ are commuting subalgebras of $C^{\infty}(P)$. In terms of coordinates, if bracket relations $\left\{x_{i}, x_{j}\right\}=w_{i j}(x)$ and $\left\{y_{i}, y_{j}\right\}=v_{i j}(y)$ are given, then these define a bracket on functions of $x$ and $y$ when augmented by the relations $\left\{x_{i}, y_{j}\right\}=0$.

The main theorem of this section states that every Poisson manifold is locally the product of a symplectic manifold and a Poisson manifold having a point where the rank is zero.

Theorem 2.1 (Splitting theorem). Let $x_{0}$ be any point in a Poisson manifold $P$. Then there are a neighborhood $U$ of $x_{0}$ in $P$ and an isomorphism $\phi=\phi_{S} \times \phi_{N}$ from $U$ to a product $S \times N$ such that $S$ is symplectic and the rank of $N$ at $\phi_{N}\left(x_{0}\right)$ is zero. The factors $S$ and $N$ are unique up to local isomorphism.

Proof. (The following existence proof for the decomposition is essentially a standard proof of Darboux's theorem for symplectic manifolds.) If the rank of $P$ at $x_{0}$ is zero, we are done; otherwise, there are functions $q_{1}^{\prime}$ and $p_{1}$ such that $\left\{q_{1}^{\prime}, p_{1}\right\}\left(x_{0}\right) \neq 0$. Then $\xi_{p_{1}}\left(x_{0}\right) \neq 0$, so by straightening out this vector field near $x_{0}$ we can find a function $q_{1}$ such that $\xi_{p_{1}} q_{1}=1$, i.e., $\left\{q_{1}, p_{1}\right\}=1$. Now the vector fields $\xi_{q_{1}}$ and $\xi_{p_{1}}$ commute, so we can find functions $x_{3}, \cdots, x_{n}$ such that the $x_{j}$ 's commute with $q_{1}$ and $p_{1}$, and $\left(q_{1}, p_{1}, x_{3}, \cdots, x_{r}\right)$ form a coordinate system. By Poisson's theorem, the brackets $\left\{x_{i}, x_{j}\right\}$ commute with $q_{1}$ and $p_{1}$ as well; thus

$$
0=\left\{\left\{x_{i}, x_{j}\right\}, q_{1}\right\}=\frac{\partial\left\{x_{i}, x_{j}\right\}}{\partial p_{1}}\left\{p_{1}, q_{1}\right\}+\text { zeros }
$$

so $\left\{x_{i}, x_{j}\right\}$ is independent of $p_{1}$ and similarly of $q_{1}$. Hence we have relations $\left\{x_{i}, x_{j}\right\}=v_{i j}(x)$, so $P$ is locally the product of a two-dimensional symplectic manifold and a Poisson manifold whose dimension and rank at each point are two less than for $P$.

Repeating this process as often as necessary, we find "canonical" coordinates $\left(q_{1}, \cdots, q_{k}, p_{1}, \cdots, p_{k}, y_{1}, \cdots, y_{s}\right)$ with $\left\{q_{i}, q_{j}\right\}=\left\{p_{i}, p_{j}\right\}=\left\{q_{i}, y_{i}\right\}=$ $\left\{p_{i}, y_{j}\right\}=0,\left\{q_{i}, p_{j}\right\}=\delta_{i j}$, and $\left\{y_{i}, y_{j}\right\}=v_{i j}(y)$ with $v_{i j}=0$ at $x_{0}$. This completes the existence proof. 
The uniqueness of the symplectic factor $S$ follows from the fact that its dimension is just the rank of $P$ at $x_{0}$. A "natural" representative for $S$ is the symplectic leaf through $x_{0}$. (In fact, the argument up to this point gives another proof of the existence of the symplectic leaf.)

To prove the uniqueness of $N$, we will identify the functions $v_{i j}(y)$ as the induced Poisson structure on a submanifold transverse to the symplectic leaf, using Proposition 1.4.

Suppose then that we have coordinates $(p, q, y)$ as just constructed. We may assume that all the coordinates vanish at $x_{0}$, so we may identify the symplectic factor $S$ with the set $y=0$ and the singular factor $N$ with $q=p=0$. We shall show that the Poisson structure on $N$ is just the induced structure from Proposition 1.4, with $N$ considered as a submanifold.

The tangent bundle $T N$ is spanned by the vector fields $\partial / \partial y_{i}$ and the orthogonal bundle $T Q^{\perp}$ by the 1-forms $d q_{j}$ and $d p_{j}$. Now $B\left(d q_{j}\right)=-\partial / \partial p_{j}$ and $B\left(d p_{j}\right)=\partial / \partial q_{j}$, so the hypothesis of Proposition 1.4 are satisfied. The projection $\pi$ from $T_{N} P$ to $T N$ kills $\partial / \partial q_{j}$ and $\partial / \partial p_{j}$ and fixes $\partial / \partial y_{i}$, so $\pi^{*}$ takes $d y_{i}$ to $d y_{i}$. Thus the induced Poisson structure maps

$$
d y_{i} \mapsto d y_{i} \mapsto \sum_{j=1}^{s} v_{i j} \frac{\partial}{\partial y_{j}} \mapsto \sum_{j=1}^{s} v_{i j} \frac{\partial}{\partial y_{j}}
$$

and is equal to the structure on the factor $N$.

Since the induced structure on a submanifold is canonically defined, our proof of the uniqueness theorem will be complete if we can prove the following lemma.

Lemma 2.2. Let $N_{0}$ and $N_{1}$ be submanifolds of the Poisson manifold $P$ having complementary dimension to a symplectic leaf $S$. Suppose that each $N_{i}$ intersects $S$ at a single point transversely. Then there is an automorphism of $P$ which maps a neighborhood of $N_{0} \cap S$ in $N_{0}$ onto a neighborhood of $N_{1} \cap S$ in $N_{1}$. (This automorphism induces an isomorphism of the induced Poisson structures on the neighborhoods.)

Proof. By integrating hamiltonian vector fields, we can map $N_{0} \cap S$ to $N_{1} \cap S$, so we may assume that these points of intersection are actually the same. Next we interpolate between $N_{0}$ and $N_{1}$ by a family of manifolds which can be defined by equations of the form $q_{j}=Q_{j}\left(y_{1}, \cdots, y_{s}, t\right)$ and $p_{j}=$ $P_{j}\left(y_{1}, \cdots, y_{s}, t\right)$, where $t \in[0,1]$, and $P_{j}$ and $Q_{j}$ are smooth functions.

We will find a family $\Psi_{t}$ of Poisson automorphisms such that $\Psi_{0}$ is the identity, and $\Psi_{t}$ maps a neighborhood of $N_{0} \cap S$ in $N_{0}$ onto $N_{t}$. The $\Psi_{t}$ are obtained by integrating a time-dependent hamiltonian vector field $\xi_{H_{i}}$. In order 
that the integral curves of $\xi_{H_{t}}$ " track" the $N_{t}$ 's, we must have the equations

$$
\begin{aligned}
& \dot{q}_{j}=\sum_{i=1}^{s} \frac{\partial Q_{j}}{\partial y_{i}} \dot{y}_{i}+\frac{\partial Q_{j}}{\partial t}, \\
& \dot{p}_{j}=\sum_{i=1}^{s} \frac{\partial P_{j}}{\partial y_{i}} \dot{y}_{i}+\frac{\partial P_{j}}{\partial t}
\end{aligned}
$$

satisfied along $N_{t}$. Substituting the coefficients of $\xi_{H_{t}}$ for $\dot{q}_{j}, \dot{p}_{j}$ and $\dot{y}_{i}$, we find

$$
\begin{aligned}
\frac{\partial H_{t}}{\partial p_{j}} & =\sum_{i, l=1}^{s} \frac{\partial Q_{j}}{\partial y_{i}} \frac{\partial H_{t}}{\partial y_{l}} v_{i l}+\frac{\partial Q_{j}}{\partial t}, \\
-\frac{\partial H_{t}}{\partial q_{j}} & =\sum_{i, l=1}^{s} \frac{\partial P_{j}}{\partial y_{i}} \frac{\partial H_{t}}{\partial y_{l}} v_{i l}+\frac{\partial P_{j}}{\partial t} .
\end{aligned}
$$

In other words, the effect on $H$ of vector fields of the form

$$
\frac{\partial}{\partial p_{j}}+\sum c_{j l} \frac{\partial}{\partial y_{l}}, \quad \frac{\partial}{\partial q_{j}}+\sum d_{j l} \frac{\partial}{\partial y_{l}}
$$

is prescribed along $N_{t}$. On $N_{t} \cap S$, the functions $v_{i l}$ and hence $c_{j l}$ and $d_{j l}$ are zero, so the vector fields are transverse to $N_{t}$, and the required function $H_{t}$ can be found. (For instance, one could begin by setting $H_{t}=0$ along $N_{t}$.) This completes the proof of the lemma and, hence, of the splitting theorem. q.e.d.

An easy corollary of the existence part of the splitting theorem is the following basic result of Lie [29] mentioned in the Introduction.

Corollary 2.3. Suppose that the rank of the Poisson manifold is constant near $x_{0}$. Then there are coordinates $\left(q_{1}, \cdots, q_{k}, p_{1}, \cdots, p_{k}, y_{1}, \cdots, y_{s}\right)$ near $x_{0}$ satisfying the canonical bracket relations $\left\{q_{i}, q_{j}\right\}=\left\{p_{i}, p_{j}\right\}=\left\{q_{i}, y_{j}\right\}=\left\{p_{i}, y_{j}\right\}=$ $\left\{y_{i}, y_{j}\right\}=0,\left\{q_{i}, p_{j}\right\}=\delta_{i j}$.

Proof. In the coordinates given by the splitting theorem, the rank of $v_{i j}(y)=\left\{y_{i}, y_{j}\right\}$ must be constant. Since it is zero at $x_{0}$, it must be identically zero. q.e.d.

As a consequence of the proof of the uniqueness part of the splitting theorem, there is a well-defined notion of "transverse Poisson structure" along any symplectic leaf: the induced Poisson structures on all cross sections to the symplectic leaf are locally isomorphic, but there is no natural representative for this transverse structure.

Another consequence of the splitting theorem is that the symplectic leaves in $P$ near $x_{0}$ are locally products of $S$ with symplectic leaves in the transverse Poisson manifold. A global conclusion may be drawn if the transverse Poisson manifold $N$ is stable near $N \cap S$ in the sense that $N$ has arbitrarily small neighborhoods of $N \cap S$ which are invariant under all hamiltonian vector 
fields. A proof of the following result can be modeled on the proof of the Reeb [38] stability theorem for foliations.

Corollary 2.4. Let $S$ be a closed simply connected symplectic leaf in $P$ such that the transverse Poisson structure is stable. Then $S$ has arbitrarily small neighborhoods in $P$ which are invariant under all hamiltonian vector fields. Each symplectic leaf of $P$ near $S$ is a bundle over $S$ whose fibre is a symplectic leaf in the transverse Poisson structure.

If $S$ is not simply connected, then we need a stronger stability condition: the transverse Poisson structure should have small neighborhoods which are invariant under all automorphisms. In this case, the symplectic leaves near $S$ are again bundles over $S$ with the fibre being a disjoint union of symplectic leaves for the transverse structure. In general (for instance, if the transverse Poisson structure is zero), there is nothing to prevent symplectic leaves from wandering apart, so that the splitting theorem gives no global information.

We note that the space of Casimir functions on a neighborhood of a point is isomorphic to the space of Casimirs for the transverse Poisson structure. The same holds in the neighborhood of a simply connected symplectic leaf when the transverse Poisson structure is stable.

We can think of a neighborhood of $S$ in $P$ as being a bundle of Poisson manifolds with fibre $N$ over the symplectic manifold $S$. It is not clear whether every such bundle arises from a Poisson manifold in which $S$ is embedded as a symplectic leaf; this is related to the question of which bundles of symplectic manifolds over a symplectic base admit a "compatible" symplectic structure on the total space. (See Weinstein [48] and Gotay, Sniatycki and Weinstein [12].)

\section{Linear Poisson structures}

A Poisson structure on a vector space $V$ is called linear if the Poisson bracket of any two linear functions on $V$ is again linear. This makes the dual space $V^{*}$ into a Lie algebra which we shall denote by $g$. $V$ is then the dual $g^{*}$ of a Lie algebra, and the Poisson bracket on $V$ is given by

$$
\{F, G\}(\mu)=\left\langle\mu,\left[\frac{\delta F}{\delta \mu}, \frac{\delta G}{\delta \mu}\right]\right\rangle,
$$

where $[$,$] is the Lie algebra operation in \mathfrak{g},\langle$,$\rangle is the pairing of \mathfrak{g}^{*}$ with $\mathfrak{g}$, and $\delta F / \delta \mu$ is the differential of $F$ considered as an element of $g$ instead of $g^{* *}$.

The formula (1) defines a linear Poisson structure on the dual space $\mathrm{g}^{*}$ of any Lie algebra $\mathrm{g}$. We shall call this the Lie-Poisson structure, since it was discovered by Lie [29] who wrote down the structure in local coordinate form. 
If $X_{1}, \cdots, X_{r}$ is a basis of $g$ satisfying $\left[X_{i}, X_{j}\right]=\sum_{k=1}^{r} c_{i j k} X_{k}$, then the $X_{i}$ considered as coordinate functions $x_{1}, \cdots, x_{r}$ on $\mathfrak{g}^{*}$ have the Poisson bracket relations $\left\{x_{i}, x_{j}\right\}=\sum_{k=1}^{r} c_{i j k} x_{k}$, i.e., the components of the Poisson structure are $w_{i j}(x)=\sum_{k=1}^{r} c_{i j k} x_{k}$. These are linear functions of $x$, hence the term "linear Poisson structure."

Let $G$ be the simply connected Lie group whose Lie algebra is $g$. The Lie algebra homomorphism $X_{i} \mapsto \xi_{x_{i}}$ from $\mathrm{g}$ to the hamiltonian vector fields on $\mathrm{g}^{*}$ induces a homomorphism from $G$ to the group of linear Poisson automorphisms of $\mathrm{g}^{*}$. This homomorphism is called the coadjoint representation, since it is dual to the adjoint representation of $G$ on g. (See Lie [29].) The coadjoint representation is trivial on the center of $G$, so in fact it is defined for any Lie group whose Lie algebra is $G$.

The hamiltonian vector field of any function $F$ on $\mathrm{g}^{*}$ is the linear combination $\xi_{F}=\sum_{i=1}^{r}\left(\partial F / \partial X_{i}\right) \xi_{x_{i}}$, so the orbits of all hamiltonian vector fields lie on orbits of the coadjoint representation. These coadjoint orbits are precisely the symplectic leaves in $\mathrm{g}^{*}$. The Casimir functions are just the functions on $\mathrm{g}^{*}$ which are invariant under the coadjoint representation (the name Casimir functions comes from this example).

For $\mu \in \mathrm{g}^{*}$, we denote its coadjoint isotropy subgroup by $G_{\mu}$, the Lie algebra of $G_{\mu}$ by $g_{\mu}$, and the coadjoint orbit by $G \cdot \mu \approx G / G_{\mu}$.

Theorem 3.1. The transverse Poisson structure at $\mu$ to the symplectic leaf $G \cdot \mu$ is isomorphic to the Lie-Poisson structure on $\mathfrak{g}_{\mu}^{*}$.

Proof. For any $\mu \in \mathrm{g}^{*}$, the map $B_{\mu}: \mathrm{g} \rightarrow \mathrm{g}^{*}$ is defined by $\left\langle B_{\mu} x, y\right\rangle=$ $-\langle\mu,[x, y]\rangle$, which also equals $\left\langle\operatorname{ad}_{x}^{*} \mu, y\right\rangle$. Then $\operatorname{Ker} B_{\mu}=\left\{x \mid \operatorname{ad}_{x}^{*} \mu=0\right\}=g_{\mu}$, and so the tangent space to the coadjoint orbit is $\operatorname{Im} B_{\mu}=\left(\operatorname{Ker} B_{\mu}\right)^{\perp}=\mathrm{g}_{\mu}^{\perp}$. Let $V \subseteq \mathrm{g}^{*}$ be a complement to $\mathrm{g}_{\mu}^{\perp}$, so that $V^{\perp}$ is a complement to $\mathrm{g}_{\mu}$ in $\mathrm{g}$. Thus the transverse Poisson structure at $\mu$ can be defined on the affine subspace $V+\mu$, which can be identified with $\mathrm{g}_{\mu}^{*}$ by the isomorphism $\alpha: \lambda+\left.\mu \mapsto \lambda\right|_{\mathbb{g}_{\mu}}$. The cotangent spaces to $V+\mu$ are then identified with $g_{\mu}$.

Now the transverse Poisson structure on $V+\mu$ at the point $\lambda+\mu$ is given by the bilinear form on $\mathrm{g}_{\mu}$

$$
\begin{aligned}
(x, y) & \mapsto\langle\lambda+\mu,[x, y]\rangle=\langle\lambda,[x, y]\rangle+\langle\mu,[x, y]\rangle \\
& =\left\langle\left.\lambda\right|_{\mathbb{g}_{\mu}},[x, y]\right\rangle+0,
\end{aligned}
$$

(since $\operatorname{ad}_{x}^{*} \mu=0$ ), which equals $\langle\alpha(\lambda+\mu),[x, y]\rangle$ and so is just the natural Poisson structure on $\mathfrak{g}_{\mu}^{*}$.

Corollary 3.2. The coadjoint orbits near a simply connected orbit $G \cdot \mu$ are bundles over $G \cdot \mu$ whose fibres are coadjoint orbits in $\mathfrak{g}_{\mu}^{*}$.

We can also derive a well-known theorem of Duflo and Vergne [8]. In fact, our Theorem 3.1 can be considered as a generalization of their result. 
Corollary 3.3. If $\mu \in \mathrm{g}^{*}$ is a regular point in $\mathrm{g}^{*}$ (i.e., a point where the rank of the Poisson structure is locally constant), then $\mathrm{g}_{\mu}$ is abelian.

Proof. The transverse Poisson structure is always trivial at a regular symplectic leaf.

\section{The linear approximation}

In view of the splitting theorem, the local study of Poisson manifolds can be reduced to the case where the rank at a point is zero. We shall see that the Poisson structure near any such point can be approximated by a linear one.

If $x$ is any point in a manifold $P$, the cotangent space $T_{x}^{*} P$ may be identified with the quotient $\mathfrak{m}_{x} / \mathfrak{m}_{x}^{2}$, where $\mathfrak{m}_{x}^{k}$ is the ideal in $C^{\infty}(P)$ consisting of all functions which vanish at $x$ together with all derivatives of order up to $k-1$. If $P$ is a Poisson manifold, then $m_{x}$ is a Lie subalgebra of $C^{\infty}(P)$. Furthermore, if the rank is zero at $x$, then $\mathrm{m}_{x}^{2}$ is a Lie ideal in $\mathrm{m}_{x}$. In fact, since $\mathrm{m}_{x}^{2}$ is generated by products $G H$ of elements in $\mathfrak{m}_{x}$, it suffices to prove that $\{F, G H\}$ is a sum of such products when $F, G$, and $H$ are in $\mathfrak{m}_{x}$. But $\{F, G H\}=$ $\{F, G\} H+\{F, H\} G$ is such a sum because all Poisson brackets are zero at $x$.

Now $T_{x}^{*} P=\mathfrak{m}_{x} / \mathfrak{m}_{x}^{2}$ has the structure of a Lie algebra, which we shall denote by $\mathfrak{g}_{x}$, and so $T_{x} P=\mathfrak{g}_{x}^{*}$ carries a Lie-Poisson structure. We call this the linear approximation to the structure at $x$. If $x_{1}, \cdots, x_{r}$ are coordinates on $P$ which all vanish at $x$, then the functions $w_{i j}=\left\{x_{i}, x_{j}\right\}$ vanish at 0 , so we can write $w_{i j}(x)=\sum_{k=1}^{r} c_{i j k} x_{k}+O\left(x^{2}\right)$ where $c_{i j k}=\partial w_{i j} / \partial x_{k}(0)$. Then the $c_{i j k}$ are the structure constants of $g_{x}$, and the coefficients of the linear approximation are just the truncations $\sum_{k=1}^{r} c_{i j k} x_{k}$ of $w_{i j}$.

The linear approximation can also be described in terms of the bundle map $B: T^{*} P \rightarrow T P$. If $B_{x}: T_{x}^{*} P \rightarrow T_{x} P$ is zero, there is a well-defined intrinsic derivative (Golubitsky and Guillemin [11]) $D_{x} B$ which is a linear map from $T_{x} P$ to $\operatorname{Hom}\left(T_{x}^{*} P, T_{x} P\right)$. Identifying the $T_{x}^{*} P$ and $T_{x} P$ inside the "Hom" with tangent and cotangent spaces to $T_{x} P$, we may consider $D_{x} B$ as a bundle map from $T^{*}\left(T_{x} P\right)$ to $T\left(T_{x} P\right)$, which is just the linear approximation Poisson structure on $T_{x} P$.

To approximate a Poisson structure at a point $x$ where the rank is not zero, we first apply the splitting theorem and then take the linear approximation to the (transverse) Poisson structure on the factor $N$. This Poisson structure lives in a natural way on the normal space $N_{x}=T_{x} P / \operatorname{Im} B_{x}$ to the symplectic leaf through $x$. One way to see this is by using the intrinsic derivative again. If $B_{x}$ is not necessarily zero, $D_{x} B$ maps $T_{x} P$ to $\operatorname{Hom}\left(\operatorname{Ker} B_{x}, T_{x} P / \operatorname{Im} B_{x}\right)$. Since $B$ is skew symmetric, $\operatorname{Ker} B_{x}=\left(\operatorname{Im} B_{x}\right)^{\perp} \approx\left(T_{x} P / \operatorname{Im} B_{x}\right)^{*}=N_{x}^{*}$, so $D_{x} B$ maps $T_{x} P$ 
to $\operatorname{Hom}\left(N_{x}^{*}, N_{x}\right)$. Now one can see easily, for instance from the local coordinate formulation of the splitting, that $D_{x} B$ annihilates the tangent space $\operatorname{Im} B_{x}$ to the symplectic leaf through $x$, so $D_{x} B$ induces a map from $N_{x}$ to $\operatorname{Hom}\left(N_{x}^{*}, N_{x}\right)$; this map is just the linear approximation to the transverse Poisson structure. The dual space $N_{x}^{*}$, called the conormal space, has the structure of a Lie algebra which we may denote by $\mathfrak{g}_{x}$ as before. As we have seen, if $P=\mathrm{g}^{*}$ with the natural Poisson structure, then $\mathrm{g}_{x}$ really is the isotropy algebra of $x$, so the notations are consistent.

Notice that the linear approximation to the transverse Poisson structure lives on a well-defined quotient space, while the transverse Poisson structure is just an equivalence class of isomorphic structures with no natural representative. If $S$ is any symplectic leaf, the linear approximations to the transverse Poisson structures make the normal bundle $N S=\cup_{x \in S} N_{x}$ into a bundle of linear Poisson manifolds, and the conormal bundle $N^{*} S$ becomes a bundle of Lie algebras.

\section{Hamiltonian systems}

If $H$ is a function on a Poisson manifold, the flow $\exp t \xi_{H}$ of the hamiltonian vector field $\xi_{H}$ leaves each symplectic leaf $S$ invariant. Since $\exp t \xi_{H}$ is a Poisson automorphism, it lifts to a bundle mapping $\phi^{t}$ from $N S$ to itself such that each mapping $N_{x} \stackrel{\phi_{x}^{t}}{\rightarrow} N_{\left(\exp t \xi_{H}\right)(x)}$ is a linear Poisson mapping. If we are given some identification of the Poisson manifolds $N_{\left(\exp t \xi_{H}\right)(x)}$ with a fixed linear Poisson manifold $N$, then the maps $\phi_{x}^{t}$ determine a time-dependent linear hamiltonian system on $N$ which is a linear approximation to the original hamiltonian system along the trajectory $\left(\exp t \xi_{H}\right)(x)$.

Suppose now that there is a symmetry for $H$, i.e., a function $K$ such that $\{K, H\}=0$, and suppose that the "dynamical" trajectory $\left(\exp t \xi_{H}\right)(x)$ coincides with the symmetry orbit $\left(\exp t \xi_{K}\right)(x)$. Then the 1-parameter group $\left(\exp t \xi_{K}\right)^{-1}\left(\exp t \xi_{H}\right)=\exp t \xi_{(H-K)}$ leaves the point $x$ fixed, so its linear approximation at $x$ gives a 1-parameter group of linear Poisson mappings on the transverse space $N_{x}=\mathfrak{g}_{x}^{*}$. The hamiltonian is obtained from the differential of $H-K$ at $x$, which annihilates $T_{x} S$ and so passes to a linear function on the quotient $N_{x}$. This linear hamiltonian may also be considered as an element of the Lie algebra $g$, and its flow is a 1-parameter subgroup of the corresponding Lie group, acting on $\mathfrak{g}^{*}$ by the coadjoint representation.

Applications of this linear approximation procedure to some mechanical systems will be given at the end of the paper. 


\section{The linearization problem}

Motivated by the linearization results for other geometric structures (vector fields, differential forms, group actions, etc.), we may ask whether a given Poisson structure near a point of rank 0 is isomorphic to its linear approximation at that point. If so, we shall say that the Poisson structure can be linearized. In other words, if a Poisson structure is of the form $w_{i j}=$ $\sum_{k=1}^{r} c_{i j k} x_{k}+O\left(x^{2}\right)$, does dropping the higher order term $O\left(x^{2}\right)$ change the qualitative nature of the Poisson structure, or can these terms be removed by a suitable change of coordinates? ${ }^{5}$ The linearization problem is the deepest and most difficult question in our study of Poisson manifolds; in fact, we can give only partial answers.

First of all, we note that as a consequence of Theorem 3.1, the Poisson structure on $\mathrm{g}^{*}$ can be linearized at every point, and the set of points at which any Poisson structure can be linearized is open. It is also dense, since the structure at any regular point can obviously be linearized.

As with other geometric structures, the answer to the linearization question depends on the nature of the linear approximation, as well as the category (formal, analytic, $C^{\infty}$ ) in which the linearization is to be carried out. ${ }^{6} \mathrm{We}$ shall call a Lie algebra $g$ (formally, analytically, $C^{\infty}$ ) nondegenerate if any Poisson manifold whose linear approximation at a point $x$ is isomorphic to $\mathrm{g}^{*}$ is itself isomorphic to $\mathrm{g}^{*}$ by a (formal, analytic, $C^{\infty}$ ) local equivalence.

It is easy to see that not every Lie algebra is nondegenerate. The Poisson structure on $\mathbf{R}^{3}$ defined by

$$
\begin{aligned}
& \left\{x_{1}, x_{2}\right\}=|x|^{2} x_{3}, \\
& \left\{x_{2}, x_{3}\right\}=|x|^{2} x_{1}, \\
& \left\{x_{3}, x_{1}\right\}=|x|^{2} x_{2}
\end{aligned}
$$

is not trivial, but its linear approximation at zero is. Similarly, one can show that no abelian Lie algebra is nondegenerate in any of our three senses.

\footnotetext{
${ }^{5}$ Note that we consistently use the term linearization to refer to the removal of higher order terms by change of coordinates, while their removal by simple truncation is called linear approximation. At a point where the rank is not zero, the linearizability will refer to the transverse Poisson structure.

${ }^{6}$ For instance, a function $\sum_{i, j=1}^{r} a_{i j} x_{i} x_{j}+O\left(x^{3}\right)$ is necessarily equivalent to its quadratic part only if the symmetric matrix $a_{i j}$ is nonsingular (Morse lemma). A differential form $\omega=$ $\sum_{i, j=1}^{r} a_{i j} x_{i} d x_{j}+0\left(x^{2}\right)$ satisfying $\omega \wedge d \omega=0$ (Pfaffian form) is necessarily of the form gdf in the formal or analytic category only if the symmetric matrix $a_{i j}$ is nonsingular. In the $C^{\infty}$ cateogry, one must add the extra condition that the index of $\left(a_{i j}\right)$ not be equal to 1 or $r-1$ (Reeb [38], Moussu [37]).
} 
On the other hand, we can show

Theorem 6.1. Any semisimple Lie algebra $\mathrm{g}$ is formally nondegenerate.

Proof. If we are given formal power series $w_{i j}\left(x_{1}, \cdots, x_{r}\right)$ with $w_{i j}(0)=0$, satisfying the antisymmetry and Jacobi identities, then they define a Lie algebra structure on the ring $\mathbf{R}\left[\left[x_{1}, \cdots, x_{\mathbf{r}}\right]\right]$ of formal power series. The powers $\mathrm{m}^{k}$ of the maximal ideal are Lie subalgebras, and $\mathfrak{m}^{2}$ is a Lie ideal in $\mathfrak{m}$, so $\mathrm{m} / \mathrm{m}^{2}$ is an $r$-dimensional Lie algebra $\mathfrak{g}$, and the Poisson manifold $\mathrm{g}^{*}$ is the linear approximation to our original formal Poisson structure.

Let $A_{k}$ be the Lie algebra $\mathrm{m} / \mathrm{m}^{k}$. Then we have a commutative diagram

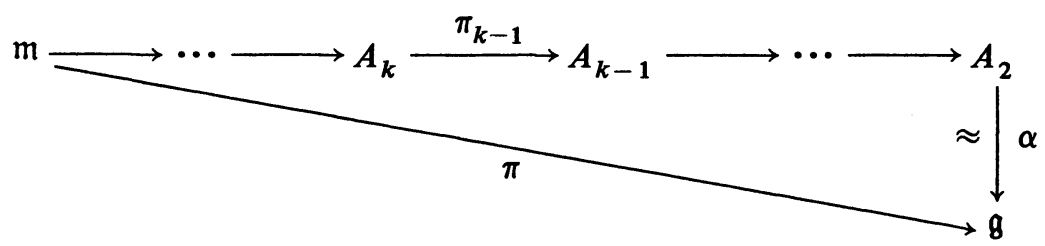

and the linearization problem is to lift the isomorphism $\alpha^{-1}$ through the tower of $A_{k}$ 's and so up to $\mathrm{m}$. In other words, we have to find formal power series $\phi_{1}, \cdots, \phi_{r}$ in $m$ such that $\left\{\phi_{i}, \phi_{j}\right\}=\sum_{k=1}^{r} c_{i j k} \phi_{k}$, where the $c_{i j k}$ 's are determined by $\left\{\pi\left(x_{i}\right), \pi\left(x_{j}\right)\right\}=\Sigma c_{i j k} \pi\left(x_{k}\right)$ in $\mathrm{g}$.

The kernels of the homomorphisms $\pi_{k-1}$ are easily seen to be abelian. Since $\mathfrak{g}$ is semisimple, all cohomology groups $H^{2}(\mathfrak{g}, V)$ are zero, and by standard results in the extension theory of Lie algebras (Jacobson [22]), the homomorphism $\alpha^{-1}$ can be lifted to each $A_{k}$ and so up to the inverse limit m. q.e.d.

We may now use Borel's theorem on the existence of $C^{\infty}$ functions with given Taylor expansions to conclude

Corollary 6.2. If $x$ is a point of rank 0 in a Poisson manifold such that the linear approximation is the dual of a semisimple Lie algebra, then there are coordinates $\left(x_{1}, \cdots, x_{r}\right)$ about $x$ and constants $c_{i j k}$ such that $\left\{x_{i}, x_{j}\right\}=\Sigma c_{i j k} x_{k}$ $+0\left(x^{\infty}\right)$.

The problem of $C^{\infty}$ nondegeneracy is also a lifting problem, from formal power series to $C^{\infty}$ functions, but now the kernel of the homomorphism (flat functions) is infinite dimensional and nonabelian so the cohomology theory does not apply. For analytic nondegeneracy, the problem is to show that the formal power series constructed in Theorem 6.1 are convergent; none of the available theory (e.g., Malgrange [30]) on analytic solutions of partial differential equations seems to apply. At this point we then must be content with some examples and conjectures.

A useful guide, especially in three dimensions, is the theory of Pfaffian forms, already mentioned in a footnote earlier in this section. Also suggestive is 
the theory of linearization of group actions (Hermann [18], Guillemin and Sternberg [14]). Study of some examples in these theories leads, in particular, to the following result.

Proposition 6.3. The semisimple algebra $\mathfrak{S} \mathfrak{I}(2, \mathbf{R})$ is not $C^{\infty}$ nondegenerate.

Proof. The Poisson structure for $S L(2, \mathbf{R})$ is given by $\left\{x_{1}, x_{2}\right\}=-x_{2}$, $\left\{x_{2}, x_{3}\right\}=x_{1},\left\{x_{3}, x_{1}\right\}=x_{2}$. The function $x_{1}^{2}+x_{2}^{2}-x_{3}^{2}$ commutes with everything, and the components of its level surfaces (cone, hyperboloids of one and two sheets) are symplectic leaves. Now we will perturb the Poisson structure to unwrap the hyperboloids of one sheet into surfaces which spiral toward the cone. To do this, we choose a $C^{\infty}$ function $l(t)$ with $l(t)=0$ for $t \leqslant 0$ and $l(t)>0$ for $t>0$, and let

$$
\begin{aligned}
& \left\{x_{1}, x_{2}\right\}=-x_{3},\left\{x_{2}, x_{3}\right\}=x_{1}-\frac{x_{2}}{x_{1}^{2}+x_{2}^{2}} l\left(x_{1}^{2}+x_{2}^{2}-x_{3}^{2}\right), \\
& \left\{x_{3}, x_{1}\right\}=x_{2}+\frac{x_{1}}{x_{1}^{2}+x_{2}^{2}} l\left(x_{1}^{2}+x_{2}^{2}-x_{3}^{2}\right) .
\end{aligned}
$$

The hamiltonian vector field $\xi_{x_{3}}$ is

$$
\left(x_{1} \frac{\partial}{\partial x_{2}}-x_{2} \frac{\partial}{\partial x_{1}}\right)-\frac{l\left(x_{1}^{2}+x_{2}^{2}-x_{3}^{2}\right)}{x_{1}^{2}+x_{2}^{2}}\left(x_{1} \frac{\partial}{\partial x_{1}}+x_{2} \frac{\partial}{\partial x_{2}}\right),
$$

whose integral curves outside the cone $x_{1}^{2}+x_{2}^{2}-x_{3}^{2}=0$ spiral in slowly toward the cone. This behavior distinguishes the given Poisson manifold from its linear approximation $\mathfrak{s} \mathfrak{l}(2, \mathbf{R}) *$.

The difficulties with $\mathfrak{I} \mathfrak{I}(2, \mathbf{R})^{*}$ seem to involve the nonsimple connectivity of the symplectic leaves. By contrast, it seems likely that one can prove the $C^{\infty}$ and analytic nondegeneracy of $\mathfrak{S} \mathfrak{o}(3)$. (By analytic continuation as in Guillemin and Sternberg [14], one could then derive the analytic nondegeneracy of $\mathfrak{S I}(2, \mathbf{R})$.) The first step would be to use the theorems of Reeb [38] and Moussu [37] to "linearize" the foliation by symplectic leaves. Next, a "volume-preserving Morse lemma" (Vey [45], Guillemin [13]) would be used to put in standard form the function which measures the symplectic area of the leaves. Finally, the deformation method of Moser [36] and Weinstein [47] would have to be applied to each symplectic leaf, with care taken to assure regularity at the origin.

We conclude this section with

Conjecture 6.4. (a) $g$ is formally or analytically nondegenerate if and only if $\mathfrak{g}$ is semisimple.

(b) If $\mathrm{g}$ is semisimple, then $\mathrm{g}$ is $C^{\infty}$ nondegenerate if and only if the index of the Killing form of $g$ is not 1 or $(\operatorname{dim} \mathfrak{g}-1)$; in particular, if $\mathfrak{g}$ is of compact type, then it is $C^{\infty}$ nondegenerate. 
These conjectures are not asserted with great conviction but are meant more as questions. In particular, the condition in the noncompact case of (b) may need to be modified.

\section{Function groups, realizations, and momentum mappings ${ }^{7}$}

A function group as defined by Lie [29] is a collection $\mathscr{F}$ of functions of the canonical variables $\left(q_{1}, \cdots, q_{n}, p_{1}, \cdots, p_{n}\right)$ such that

(i) $\mathscr{F}$ is a Lie algebra under Poisson bracket,

(ii) if $F_{1}, \cdots, F_{s} \in \mathscr{F}$ and $G: \mathbf{R}^{s} \rightarrow \mathbf{R}$, then $G\left(F_{1}, \cdots, F_{s}\right) \in \mathscr{F}$. Lie always assumed that $\mathscr{F}$ was generated (in the sense of the functional composition operation (ii)) by a finite number $\phi_{1}, \cdots, \phi_{r}$ of independent functions, so that (i) implies $\left\{\phi_{i}, \phi_{j}\right\}=w_{i j}\left(\phi_{1}, \cdots, \phi_{r}\right)$ for functions $w_{i j}$ on $\mathbf{R}^{r}$. Given a function group with such generators, the Jacobi identity for the canonical Poisson bracket implies that the $w_{i j}$ define a Poisson structure on $\mathbf{R}^{r}$ for which the mapping $\left(\phi_{1}, \cdots, \phi_{r}\right): \mathbf{R}^{2 n} \rightarrow \mathbf{R}^{r}$ is a Poisson mapping. Lie assumed implicitly that this Poisson structure had constant rank.

The realization problem in local terms may then be stated as follows: given a Poisson structure on an open subset $\mathcal{Q}$ of $\mathbf{R}^{r}$, find a Poisson mapping from the canonical $\mathbf{R}^{2 n}$ onto $\mathcal{Q}$ for some $n$. We will call such a mapping a realization of the Poisson structure. ${ }^{8}$ In the case of constant rank, Lie proved that every Poisson structure has a realization, and classified the realizations up to canonical transformations.

In global terms, we may define a function group by specifying an equivalence relation, or foliation, $\Phi$ on a symplectic manifold $(S, \Omega)$ such that the quotient space $S / \Omega$ is a manifold, and letting $\mathscr{F}_{\Phi}$ be the space of functions constant on the leaves (equivalence classes) of $\Phi$. The condition that $\mathscr{F}_{\Phi}$ be closed under Poisson bracket can then be expressed geometrically in the following way. Let $T \Phi \subseteq T S$ be the subbundle of vectors tangent to the leaves of $\Phi$, and let $T \Phi^{\perp}$ be its orthogonal complement under the symplectic structure $\Omega$. If $F \in \mathscr{F}_{\Phi}$ and $v \in T \Phi$, then $\Omega\left(v, \xi_{F}\right)=v \cdot F=0$, so the hamiltonian vector field $\xi_{F}$ lies in $T \Phi^{\perp}$. By dimension counting one sees now that $T \Phi^{\perp}$ is filled at each point by the hamiltonian vector fields of functions in $\mathscr{F}_{\Phi}$. If $\mathscr{F}_{\Phi}$ is a Lie algebra, then these hamiltonian vector fields are closed under

\footnotetext{
${ }^{7}$ Most of this section is essentially a reworking of Lie [29]. See also Hermann [20].

${ }^{8}$ In Marsden and Weinstein [32] and Marsden, Ratiu and Weinstein [33], the coordinates on $\mathbf{R}^{2 n}$ are called Clebsch variables for the Poisson manifold $U$. An infinite-dimensional version is studied in those papers.
} 
bracket, so the bundle $T \Phi^{\perp}$ is involutive and so is tangent to another foliation which we may call $\Phi^{\perp}$. On the other hand, if we know $T \Phi^{\perp}$ to be involutive, then the Poisson bracket of any two functions in $\mathscr{F}_{\Phi}$ has its hamiltonian vector field in $T \Phi^{\perp}$ and hence lies in $\mathscr{F}_{\Phi}$. Then we have proven

Proposition 7.1. Let $\Phi$ be a foliation on a symplectic manifold $S$. Then the space ${ }^{9} \mathscr{F}_{\Phi}$ of functions along the leaves of $\Phi$ is a function group if and only if the subbundle $T \Phi^{\perp}$ symplectically orthogonal to the leaves of $\Phi$ is involutive.

If the hypotheses of Proposition 7.1 are satisfied, the functions constant along the leaves of $\Phi^{\perp}$ form another function group $\mathscr{F}_{\Phi^{\perp}}$, which Lie called the polar group of $\mathscr{F}_{\Phi}$. Thus function groups always come in pairs. The quotient spaces $S / \Phi$ and $S / \Phi^{\perp}$ are Poisson manifolds, and in $\S 8$ we shall show that their Poisson structures are very similar to one another.

It is easily seen that the polar function group $\mathscr{F}_{\Phi^{\perp}}$ consists precisely of the functions which Poisson commute with all the elements of $\mathscr{F}_{\Phi}$; this, in fact, is the condition by which Lie defined polarity. The intersection $\mathscr{F}_{\boldsymbol{\Phi}} \cap \mathscr{F}_{\Phi^{\perp}}$ is thus equal to the center of either of the Lie algebras $\mathscr{F}_{\Phi}$ and $\mathscr{F}_{\Phi^{\perp}}$. Lie called the elements of $\mathscr{F}_{\Phi} \cap \mathscr{F}_{\Phi^{\perp}}$ the distinguished functions of $\mathscr{F}_{\Phi}$ (or $\mathscr{F}_{\Phi^{\perp}}$ ). He also observed that $\mathscr{F}_{\Phi} \cap \mathscr{F}_{\Phi^{\perp}}$ forms a new commutative function group, but here the assumption of constant rank of the Poisson manifold $S / \Phi$ must be invoked to assure that $\mathscr{F} \cap \mathscr{F}_{\Phi^{\perp}}$ is associated with a smooth foliation. As functions on the Poisson manifold $S / \Phi$, the distinguished functions are just the center of the Poisson algebra $C^{\infty}(S / \Phi)$, i.e., the Casimir functions.

A realization of the Poisson manifold $P$ is defined to be a Poisson mapping $J$ from a symplectic manifold $S$ to $P$; the realization will be called full if $J$ is a submersion. By Lemma 1.2, hamiltonian flows on $P$ can be put in canonical form with the aid of realizations; this is the essential idea behind the use of Clebsch variables in continuum mechanics.

A simple example of a realization is the inclusion map of any symplectic leaf, but this realization is generally not full. We shall show in $\$ 9$ that full realizations always exist locally, and that they are essentially unique.

A realization $S \stackrel{J}{\rightarrow} \mathrm{g}^{*}$, where $\mathrm{g}^{*}$ has its Lie-Poisson structure, corresponds to a Lie algebra homomorphism from $g$ to $C^{\infty}(S)$ and hence to a hamiltonian action on $S$ of the corresponding Lie group $G$. In this context, the map $J$ is called the momentum mapping of the group action. Since the linear functions on $\mathrm{g}^{*}$ generate the coadjoint action of $G$, it follows from Lemma 1.2 that the momentum mapping is $G$-equivariant with respect to the coadjoint action, a

\footnotetext{
${ }^{9}$ One should speak of a sheaf if the quotient space $S / \Phi$ is not a manifold.
} 
fact known already to Lie [29, Chapter 19] and rediscovered in the mid 1960's by Kostant [26] and Souriau [41].

\section{Dual pairs and gauge groups}

If $S$ is a symplectic manifold, we call a pair of Poisson mappings $P_{1} \stackrel{J_{1}}{\leftarrow} \stackrel{J_{2}}{\rightarrow} P_{2}$ a dual pair ${ }^{10}$ if the function groups $\mathscr{F}_{1}=J_{1}^{*}\left(C^{\infty}\left(P_{1}\right)\right)$ and $\mathscr{F}_{2}=J_{2}^{*}\left(C^{\infty}\left(P_{2}\right)\right)$ are polar to one another. We call the dual pair full if $J_{1}$ and $J_{2}$ are submersions onto $P_{1}$ and $P_{2}$. A dual pair may also be defined as one for which the subbundles $\operatorname{Ker} T J_{1}$ and $\operatorname{Ker} T J_{2}$ tangent to the level manifolds of $J_{1}$ and $J_{2}$ are symplectic orthogonal complements of one another.

If the maps $J_{1}$ and $J_{2}$ have constant rank, then the images $J_{1}\left(P_{1}\right)$ and $J_{2}\left(P_{2}\right)$ are Poisson submanifolds, and $J_{1}\left(P_{1}\right) \stackrel{J_{1}}{\leftarrow} S \stackrel{J_{2}}{\rightarrow} J_{2}\left(P_{2}\right)$ is a full dual pair, so for many purposes we can restrict our attention to this case.

Given a full dual pair, the spaces of Casimir functions on $P_{1}$ and $P_{2}$ are both in 1-1 correspondence with the space of distinguished functions in the polar function groups $\mathscr{F}_{1}$ and $\mathscr{F}_{2}$, so $P_{1}$ and $P_{2}$ have the "same" Casimir functions. We also note that for $x_{1} \in P_{1}$ each connected component of $J^{-1}\left(x_{1}\right)$ maps under $J_{2}$ to a symplectic leaf in $P_{2}$, and vice versa. This gives a correspondence between symplectic leaves in $P_{1}$ and $P_{2}$, which is bijective locally (and globally if $J_{1}$ and $J_{2}$ have connected fibres).

These observations, which have already been made in a purely symplectic context by Kazhdan, Kostant, and Sternberg [23] and Guillemin and Sternberg [15], suggest that the Poisson manifolds occurring in a full dual pair are very closely related. This we shall now make precise and prove.

Theorem 8.1. Let $P_{1} \stackrel{J_{1}}{\leftarrow} S \stackrel{J_{2}}{\rightarrow} P_{2}$ be a full dual pair. For each $x \in S$, the transverse Poisson structures on $P_{1}$ and $P_{2}$ at $J_{1}(x)$ and $J_{2}(x)$ are anti-isomorphic (i.e., isomorphic up to a reversal of sign of the bracket). Consequently, if $\operatorname{dim} P_{1} \leqslant \operatorname{dim} P_{2}, P_{2}$ is locally anti-isomorphic to the product of $P_{1}$ with $a$ symplectic manifold.

Proof. We begin by reducing the problem to the case where $P_{1}$ and $P_{2}$ have rank zero at $J_{1}(x)$ and $J_{2}(x)$. To accomplish this, let $\left(q_{1}, \cdots, q_{k}, p_{1}, \cdots, p_{k}\right.$, $\left.y_{1}, \cdots, y_{s}\right)$ be the local coordinates on $P_{1}$ near $J_{1}(x)$ obtained from the splitting theorem (Theorem 2.1), and let $\left(q_{1}^{\prime}, \cdots, q_{l}^{\prime}, p_{1}^{\prime}, \cdots, p_{l}^{\prime}, y_{1}^{\prime}, \cdots, y_{t}^{\prime}\right)$ be such coordinates on $P_{2}$ near $J_{2}(x)$. Pulling back via $J_{1}$ and $J_{2}$, we can consider all these coordinates as functions on $S$.

\footnotetext{
${ }^{10}$ The term dual pair was introduced in representation theory by Roger Howe in an unpublished manuscript. We are dealing here with the "classical" analog of Howe's "quantum" notion.
} 
Now $\left(q_{1}, \cdots, q_{k}, q_{1}^{\prime}, \cdots, q_{l}^{\prime}, p_{1}, \cdots, p_{k}, p_{1}^{\prime}, \cdots, p_{l}^{\prime}\right)$ satisfy the canonical commutation relations, and so they are functionally independent. By the method used in the proof of Theorem 2.1, we can find further functions $q_{1}^{\prime \prime}, \cdots, q_{m}^{\prime \prime}$, $p_{1}^{\prime \prime}, \cdots, p_{m}^{\prime \prime}$ such that $\left(q, p, q^{\prime}, p^{\prime}, q^{\prime \prime}, p^{\prime \prime}\right)$ is a canonical coordinate system on $S$. Since the $y$ 's and $y^{\prime \prime}$ s commute with $\left(q, p, q^{\prime}, p^{\prime}\right)$, they must be functions of $\left(q^{\prime \prime}, p^{\prime \prime}\right)$ alone. Thus we have split $S$ as a product $S^{\prime} \times S^{\prime \prime}$ such that the transverse parts of the Poisson structures on $P_{1}$ and $P_{2}$ really live on $S^{\prime \prime}$. It is easy to check that these transverse parts form a full dual pair on $S^{\prime \prime}$, so our reduction is complete. Thus we assume at this point that $P_{1}$ and $P_{2}$ have rank zero at $J_{1}(x)$ and $J_{2}(x)$.

We continue to use the coordinate functions $\left(y_{1}, \cdots, y_{s}\right)$ and $\left(y_{1}^{\prime}, \cdots, y_{t}^{\prime}\right)$, which we may take to vanish at $J_{1}(x)$ and $J_{2}(x)$ respectively. The conditions $\left\{y_{i}, y_{j}\right\}(x)=0$ and $\left\{y_{i}^{\prime}, y_{j}^{\prime}\right\}(x)=0$ imply that the tangent spaces $\operatorname{Ker} T_{x} J_{1}$ and $\operatorname{Ker} T_{x} J_{2}$ to the level manifolds of $J_{1}$ and $J_{2}$ through $x$ are coisotropic. By duality, these tangent spaces are orthogonal complements of one another, so they must in fact be lagrangian and equal. In particular, $t=\operatorname{dim} P_{1}=\operatorname{dim} P_{2}$ $=s$, and $\operatorname{dim} S=2 s$.

Choose a lagrangian submanifold $\Lambda \subseteq S$ through $x$ which is transverse to $\operatorname{Ker} T_{x} J_{1}=\operatorname{Ker} T_{x} J_{2}$. The functions $y_{1}, \cdots, y_{s}$ restricted to $\Lambda$ are independent and may be extended to commuting functions $q_{1}, \cdots, q_{s}$ (not the $q$ 's above) near $\Lambda$ in $S$. Next we can find functions $p_{1}, \cdots, p_{s}$ vanishing on $\Lambda$ such that $\left(q_{1}, \cdots, q_{s}, p_{1}, \cdots, p_{s}\right)$ forms a canonical coordinate system.

We may write $y_{i}=q_{i}+\sum a_{j l} p_{l}$ where the $a_{j l}$ 's are functions on $S$. By choosing coordinates on $P_{2}$, we can arrange it so that $y_{i}^{\prime}=q_{i}$ on $\Lambda$ as well, so that $y_{i}^{\prime}=q_{i}+\Sigma b_{j l} p_{l}$. Now

$$
\left\{y_{i}, y_{j}\right\}=\left\{q_{i}+\sum a_{i k} p_{k}, q_{j}+\sum a_{i l} p_{l}\right\},
$$

and so $\left\{y_{i}, y_{j}\right\}=a_{j i}-a_{i j}$ on $\Lambda$. Thus if we set $\left\{y_{i}, y_{j}\right\}=w_{i j}$, we have $w_{i j}=a_{j i}-a_{i j}$ (identifying $P_{1}$ with $\Lambda$ via $J_{1}$ ). Similarly, $\left\{y_{i}^{\prime}, y_{j}^{\prime}\right\}=w_{i j}^{\prime}$ implies $w_{i j}^{\prime}=b_{j i}-b_{i j}$. Finally the duality gives

$$
0=\left\{a_{i}, \beta_{j}\right\}=\left\{q_{i}+\sum a_{i k} p_{k}, q_{j}+\sum b_{j k} p_{k}\right\},
$$

which on $\Lambda$ gives $a_{k i}-b_{i k}=0$. Then $w_{i j}=a_{j i}-a_{i j}=b_{i j}-b_{j i}=w_{i j}^{\prime}$, so the identifications of $P_{1}$ and $P_{2}$ with $\Lambda$ give an anti-isomorphism between their Poisson structures. q.e.d.

An important way to realize Poisson manifolds is as quotients of symplectic manifolds by group actions. Suppose that a Lie group $G$ acts freely on a symplectic manifold $S$ with momentum map $J: S \rightarrow \mathrm{g}^{*}$, and that the quotient space $S / G$ is a manifold. Then the functions on $S / G$ may be identified with 
the function group of $G$-invariant functions, so $S / G$ has a Poisson structure for which the projection $\pi: S \rightarrow S / G$ is a Poisson mapping. Since the $\pi$-level manifolds are $G$ orbits, it is immediate that $\mathrm{g}^{*} \stackrel{J}{\leftarrow} S \stackrel{\pi}{\rightarrow} S / G$ is a dual pair. The symplectic leaves in $S / G$ are the manifolds $\pi\left(J^{-1}(G \cdot \mu)\right)=J^{-1}(G \cdot \mu) G$, called the reduced manifolds of $S$ by the $G$-action. (They are isomorphic to the reduced manifolds $J^{-1}(\mu) / G_{\mu}$ introduced by Marsden and Weinstein [31]; see Kazhdan, Kostant, and Sternberg [23]).

In physical applications, the symplectic manifold $S$ may represent a collection of states where elements of $S$ in the same $G$-orbit are considered to be physically indistinguishable. Thus the set of "true physical states" is the Poisson manifold $S / G$, and the group $G$ is called a gauge group. Often a Poisson manifold $P$ of physical states is given in advance, and one seeks to realize $P$ as a quotient $S / G$.

The point to be made here is that the gauge group $G$ is essentially determined by the transverse Poisson structures in $P$. By Theorem 8.1 , if $y$ is any element of $P=S / G$ with $y=\pi(x)$, then the isotropy algebra $\mathfrak{g}_{J(x)} \subseteq \mathfrak{g}$ must be such that the Lie-Poisson structure on $\mathrm{g}_{J(x)}^{*}$ is the normal Poisson structure for $P$ at $y$. In particular, only linearizable Poisson manifolds can be realized as quotients of symplectic manifolds by Lie group actions, and only regular Poisson manifolds can be realized with abelian gauge groups.

Some examples of dual pairs in mechanics may be found in Marsden and Weinstein [32] and Marsden, Ratiu and Weinstein [33].

\section{Existence of realizations}

The most important realization of a Lie-Poisson manifold $\mathrm{g}^{*}$ is by the momentum mapping $\mathrm{g}^{*} \stackrel{J}{\rightarrow} T^{*} G$ associated with the action of the corresponding Lie group $G$ on $T^{*} G$ by the lifts of left translations. $J$ is just the operation of right translation to the cotangent space at the identity element of $G$. The quotient $T^{*} G / G$ can again be identified with $g^{*}$ but with the negative of the usual Poisson structure, and the projection $T^{*} G \rightarrow \mathrm{g}^{*}$ is given by left translation. Thus we have the dual pair $\mathrm{g}^{*} \stackrel{J}{\leftarrow} T^{*} G \stackrel{\pi}{\rightarrow} \mathrm{g}^{*}$, in which the roles of $J$ and $\pi$ are completely symmetric; $\pi$ is the momentum mapping for the action of $G$ by the lifts of right translations, and $J$ is the quotient mapping for that action.

By the splitting theorem, we can use the basic example just described to find a (local) realization of any Poisson structure which is linearizable. Of course, this assumes the existence of the Lie group $G$ whose Lie algebra is $g$. But linearization at a regular element of any Poisson manifold (including an 
arbitrary $\mathrm{g}^{*}$ ) produces an abelian Lie algebra, and in this case the existence of a group is obvious; this idea was used by Lie [29] to prove the existence of a local Lie group of diffeomorphisms corresponding to any given Lie algebra.

For a given Poisson manifold which may not be linearizable, constructing a realization is tantamount to finding a substitute for the corresponding Lie group. This is the idea behind the proof of the following theorem.

Theorem 9.1. If $x$ is any point in a Poisson manifold $P$, there is a full realization of $a$ neighborhood of $x$ by a symplectic manifold of dimension 2(dim $\left.P-\frac{1}{2} \operatorname{rank}_{x} P\right)$.

Proof. By the splitting theorem, we can reduce the problem to the case where the rank of $P$ at $x$ is zero, which we shall now assume. Let $x_{1}, \cdots, x_{n}$ be a coordinate system centered at $x$, so that $\left\{x_{i}, x_{j}\right\}=w_{i j}\left(x_{1}, \cdots, x_{n}\right)$ with $w_{i j}(0)$ $=0$. We shall take the symplectic manifold $S$ to be $\mathbf{R}^{2 n}$ with coordinates $\left(x_{1}, \cdots, x_{n}, y_{1}, \cdots, y_{n}\right)$ and the obvious projection of $S$ onto $P$, but with a symplectic structure to be chosen in order that the Poisson bracket relations $\left\{x_{i}, x_{j}\right\}=w_{i j}(x)$ hold in $S$.

The manifold $x=0$ will be lagrangian, so it will be transversal to some lagrangian foliation; thus there is no less of generality in assuming that the required symplectic structure be of the form $d \phi$, where $\phi=\sum_{i=1}^{n} \phi_{i}(x, y) d y_{i}$.

With $\phi=\sum_{i=1}^{n} \phi_{i}(x, y) d y_{i}$, we have

$$
\Omega=d \phi=\sum_{i, j=1}^{n} a_{i j} d x_{i} \wedge d y_{j}+\frac{1}{2} \sum_{i, j=1}^{n} b_{i j} d y_{i} \wedge d y_{j}
$$

where $a_{i j}=\partial \phi_{j} / \partial x_{i}$ and $b_{i j}=\partial \phi_{j} / \partial y_{i}-\partial \phi_{i} / \partial y_{j}$. Then the map $\tilde{\Omega}$ from tangent vectors to cotangent vectors is given by

$$
\tilde{\Omega}\left(\frac{\partial}{\partial x_{i}}\right)=\sum_{j=1}^{n} a_{i j} d y_{j}, \quad \tilde{\Omega}\left(\frac{\partial}{\partial y_{i}}\right)=-\sum_{j=1}^{n} a_{j i} d x_{j}+\sum_{j=1}^{n} b_{i j} d y_{j} .
$$

The Poisson bracket $\left\{x_{i}, x_{j}\right\}$ is $\tilde{\Omega}^{-1}\left(d x_{j}\right) \cdot d x_{i}$. The inverse of the block matrix

$$
\left(\begin{array}{cc}
0 & -a^{t} \\
a & b
\end{array}\right)
$$

has $p=a^{-1} b\left(a^{t}\right)^{-1}$ in the upper left-hand corner, so $\left\{x_{i}, x_{j}\right\}=-p_{i j}$. Thus $-a^{-1} b\left(a^{t}\right)^{-1}=w$, where $w_{i j}$ was the given value of $\left\{x_{i}, x_{j}\right\}$ in the Poisson structure. Finally, we have the equation $b=-a w a^{t}$ which is a system of $\frac{1}{2} n(n-1)$ first-order partial differential equations for the unknown functions $\phi_{i}(x, y)$. In coordinates, we have

$$
\frac{\partial \phi_{j}}{\partial y_{i}}-\frac{\partial \phi_{i}}{\partial y_{j}}=-\sum_{\alpha, \beta} \frac{\partial \phi_{i}}{\partial x_{\alpha}} w_{\alpha \beta} \frac{\partial \phi_{j}}{\partial x_{\beta}} .
$$


Let us think of the $\phi_{i}$ 's not as scalar functions of $x$ and $y$, but rather as functions of $y$ with values in the Lie algebra $C^{\infty}(P)$ of functions of $x .^{11}$

The form $\phi=\sum \phi_{i} d y_{i}$ is then a $C^{\infty}(P)$-valued 1-form in $y$, and (1) is the component form of the equation

$$
d \phi=-\frac{1}{2}\{\phi, \phi\},
$$

where the bracket is the standard bracket on Lie algebra-valued 1-forms, as in Bishop and Crittenden [7].

We may recognize (2) as the structure equation for the Maurer-Cartan form $\omega$ on the group $\mathcal{G}$ whose Lie algebra is $C^{\infty}(P)$, or alternately as the equation for the connection form of a flat connection on a principal $\mathcal{G}$-bundle over $y$ space. Such a connection form can be found in the form $\gamma^{*} \omega$, where $\gamma$ is a map from $y$-space to $\mathcal{G}$.

Now we do not have the group $\mathcal{G}$ at our disposal, but fortunately there is a formula for the pullback of $\omega$ to the Lie algebra $C^{\infty}(P)$ under the exponential map, which is expressible purely in terms of the Lie algebra and makes sense in the present context. Namely, ${ }^{12}$

$$
\exp ^{*}(\omega)(f)=\int_{0}^{1} \exp (-s \cdot \operatorname{ad} f) d s .
$$

Here $f$ is an element of the Lie algebra $C^{\infty}(P)$, ad $f$ is the map $g \mapsto\{f, g\}$, and hence $\exp (-s \cdot \operatorname{ad} f)$ can be interpreted as the time $s$ map $\Psi^{s}$ of the hamiltonian flow $\xi_{f}$ on $P$ generated by $f$. Now if we identify each element of $y$-space with the linear function $f_{y}(x)=\sum_{i=1} x_{i} y_{i}$, we have constructed the form $\phi$ as $\Sigma \phi_{i} d y_{i}$, where

$$
\phi_{i}(x, y)=\int_{0}^{1} x_{i} \circ \Psi_{y}^{s} d s .
$$

(We write $\Psi_{y}^{s}$ for the flow of $\xi_{f_{y}}$.) In other words, $\phi_{i}$ is the average value of $x_{i}$ over the trajectory from $s=0$ to 1 of the hamiltonian flow generated by $f_{y}$.

To complete the proof, we shall verify directly that the functions $\phi_{i}$ defined by (4) satisfy the differential equations (1) and incidentally that the matrix $a_{i j}=\partial \phi_{j} / \partial x_{i}$ is nonsingular so that $d \phi$ really is a symplectic structure. We will also see explicitly that the Jacobi identity for the Poisson structure must be used, although (4) makes sense without it.

We recall the "variation of parameters" formula for the derivative of a flow with respect to the generating vector field. If $\xi$ and $\eta$ are vector fields on a

\footnotetext{
"Arguments involving this infinite-dimensional Lie algebra may be thought of as merely heuristic, since in the end we will produce an explicit formula which will be verified directly.

${ }^{12}$ The right-hand side of (3) is usually written in the symbolic form $\left(1-e^{-s \text { ad } f}\right) /(s$ ad $f)$. See Helgason [16] or Freudenthal and de Vries [9].
} 
manifold, then

$$
\frac{\partial}{\partial \varepsilon} \exp [s(\xi+\varepsilon \eta)] \mid=\exp (s \xi) \int_{0}^{s} \exp (-t \xi) \eta \exp (t \xi) d t .
$$

(See Abraham and Robbin [2, 32.1].) Here, as above, we are thinking of the vector fields and their flows as operators on functions.

Now we differentiate (4) with respect to $y_{j}$. Since $\left(\partial / \partial y_{j}\right) \xi_{f_{y}}=\xi_{x_{j}}$, we get from (5),

$$
\begin{aligned}
\frac{\partial \phi_{i}}{\partial y_{j}} & =\int_{0}^{1} \frac{\partial}{\partial y_{j}} \exp \left(s \xi_{f_{y}}\right) x_{i} d s \\
& =\int_{0}^{1} \exp \left(s \xi_{f_{y}}\right) \int_{0}^{s} \exp \left(-t \xi_{f_{y}}\right) \xi_{x_{j}} \exp \left(t \xi_{f_{y}}\right) x_{i} d t d s \\
& =\iint_{0 \leqslant t \leqslant s \leqslant 1}\left\{x_{i} \circ \Psi_{y}^{t}, x_{j}\right\} \circ \Psi_{y}^{s-t} d t d s
\end{aligned}
$$

Now the Jacobi identity implies that $\Psi_{y}^{t}$ preserves Poisson brackets, so

$$
\begin{aligned}
\frac{\partial \phi_{i}}{\partial y_{j}} & =\iint_{0 \leqslant t \leqslant s \leqslant 1}\left\{x_{i} \circ \Psi_{y}^{s}, x_{j} \circ \Psi_{y}^{s-t}\right\} d t d s \\
& =\iint_{0 \leqslant \tau \leqslant s \leqslant 1}\left\{x_{i} \circ \Psi_{y}^{s}, x_{j} \circ \Psi_{y}^{t}\right\} d \tau d s .
\end{aligned}
$$

Interchanging $i$ and $j$, we get

$$
\begin{aligned}
\frac{\partial \phi_{j}}{\partial y_{i}} & =\iint_{0 \leqslant \tau \leqslant s \leqslant 1}\left\{x_{j} \circ \Psi_{y}^{s}, x_{i} \circ \Psi_{y}^{\tau}\right\} d \tau d s \\
& =-\iint_{0 \leqslant \tau \leqslant s \leqslant 1}\left\{x_{i} \circ \Psi_{y}^{\tau}, x_{j} \circ \Psi_{y}^{s}\right\} d \tau d s \\
& =-\iint_{0 \leqslant s \leqslant \tau \leqslant 1}\left\{x_{i} \circ \Psi_{y}^{s}, x_{j} \circ \Psi_{y}^{\tau}\right\} d \tau d s,
\end{aligned}
$$

where in the last step we simply interchanged the dummy indices $s$ and $\tau$. We combine the two integrals to get an integral over the unit square

$$
\begin{aligned}
\frac{\partial \phi_{j}}{\partial y_{i}}-\frac{\partial \phi_{i}}{\partial y_{j}} & =-\iint_{0 \leqslant s, \tau \leqslant 1}\left\{x_{i} \circ \Psi_{y}^{s}, x_{j} \circ \Psi_{y}^{\tau}\right\} d \tau d s \\
& =-\left\{\int_{0}^{1}\left(x_{i} \circ \Psi_{y}^{s}\right) d s, \int_{0}^{1}\left(x_{j} \circ \Psi_{y}^{\tau}\right) d \tau\right\} \\
& =-\left\{\phi, \phi_{j}\right\}=-\sum \frac{\partial \phi_{i}}{\partial x_{r}} w_{r s} \frac{\partial \phi_{j}}{\partial x_{s}}
\end{aligned}
$$

which establishes (1). 
Finally, we note that, for $y=0, \Psi_{y}^{s}$ is the identity for all $s$, so $\phi_{i}(x, 0)=x_{i}$, and hence the matrix $a_{i j}=\partial \phi_{j} / \partial x_{i}$ is invertible in a neighborhood of $y=0$. q.e.d.

Some remarks on the proof may be of interest. First of all, since $\Omega=$ $d\left(\sum \phi_{i} d y_{i}\right)=\sum d \phi_{i} \wedge d y_{i}$, the functions $\left(\phi_{1}(x, y), \cdots, \phi_{n}(x, y), y_{1}, \cdots, y_{n}\right)$ are canonical coordinates on $S$. Thus to solve the problem of realizing the bracket relations $\left\{x_{i}, x_{j}\right\}=w_{i j}(x)$ in terms of canonical variables, it suffices to invert the transformation $(x, y) \mapsto(\phi(x, y), y)$ and solve for $x$ in terms of $\phi$ and $y$.

Next we note a special case. If the given Poisson structure is abelian, then all the transformations $\Psi_{y}^{t}$ are the identity, and so we get the obvious realization with $\phi_{i}=x_{i}$. More generally, if the Poisson structure is linear, then the transformations $\Psi_{y}^{t}$ are linear, so the functions $\phi_{i}(x, y)$ are linear in $x$. What we have obtained then is the usual local expression for the symplectic structure on the cotangent bundle of a Lie group in terms of the trivialization by left-invariant 1-forms, the $y_{i}$ 's being coordinates on the group. For a general Poisson structure, the $y_{i}$ 's are coordinates on a submanifold (but not subgroup) of the "group" whose Lie algebra is $C^{\infty}(P)$.

We saw in the beginning of this section section that every Lie-Poisson manifold $\mathrm{g}^{*}$ has a full realization $T^{*} G \stackrel{J}{\rightarrow} \mathrm{g}^{*}$, and it is easy to see that this is the "minimal" possible realization of $\mathrm{g}^{*}$ since the rank at the origin is zero. However, if we are working near a nonzero point $\mu \in \mathrm{g}^{*}$, a full realization is possible, according to Theorem 9.1 , by a symplectic manifold of dimension $2\left(\operatorname{dim} \mathfrak{g}-\frac{1}{2}\left(\operatorname{dim} \mathfrak{g}-\operatorname{dim} \mathfrak{g}_{\mu}\right)\right)=\operatorname{dim} \mathfrak{g}+\operatorname{dim} \mathfrak{g}_{\mu}$. An explicit construction of this realization may sometimes be made as follows. We need to find a free action of $G$ on a smaller manifold than $T^{*} G$, so it is natural to look for a symplectic submanifold invariant under left translations. Let $\pi: T^{*} G \rightarrow \mathrm{g}^{*}$ be the left translation map, and let $W \subseteq \mathrm{g}^{*}$ be a piece of submanifold through $\mu$ whose tangent space is complementary to the tangent space to the coadjoint orbit (i.e., $\mathrm{g}_{\mu}^{\perp}$ ). Now $\pi^{-1}(W)$ is a left-invariant submanifold of $T^{*} G$, and one can check the following:

(i) $\pi^{-1}(W)$ is symplectic near $\pi^{-1}(\mu)$.

(ii) The momentum mapping for the action of $G$ on $\pi^{-1}(W)$ by left translations is just $J \mid W$, so the image contains $\mu$.

Since the $G$ action is free, the momentum map $J \mid W$ is a submersion, and hence we have a full realization of a neighborhood of $\mu$.

An application of this construction in fluid dynamics is given in Marsden and Weinstein [32]. 


\section{Uniqueness of realizations}

The problem of uniqueness of realizations was already posed and solved in a special case by Lie [29]. Given $r$-tuples of functions $\left(\alpha_{1}, \cdots, \alpha_{r}\right)$ and $\left(\beta_{1}, \cdots, \beta_{r}\right)$ of the canonical coordinates $\left(q_{1}, \cdots, q_{n}, p_{1}, \cdots, p_{n}\right)$ on $\mathbf{R}^{2 n}$, when is there a canonical transformation $\phi$ on $\mathbf{R}^{n}$ with $\alpha_{i} \circ \phi=\beta_{i}$ ? If $\left(\alpha_{1}, \cdots, \alpha_{r}\right)$ and $\left(\beta_{1}, \cdots, \beta_{r}\right)$ each generate a function group, then an obvious necessary condition for the existence of $\phi$ is that the "structure functions" $w_{i j}$ and $v_{i j}$ be equal, where $\left\{\alpha_{i}, \alpha_{j}\right\}=w_{i j}\left(\alpha_{1}, \cdots, \alpha_{r}\right)$ and $\left(\beta_{i}, \beta_{j}\right)=v_{i j}\left(\beta_{1}, \cdots, \beta_{r}\right)$. Under the assumption that the matrices $w_{i j}$ and $v_{i j}$ have constant rank, Lie proved that this necessary condition is also sufficient. He also extended the result to the case where $\left(\alpha_{1}, \cdots, \alpha_{r}\right)$ and $\left(\beta_{1}, \cdots, \beta_{r}\right)$ are defined on spaces of different dimensions. The following theorem is an extension of Lie's result to the case of variable rank. The proof is related to an argument in Roels and Weinstein [39].

Theorem 10.1. Let $S_{1} \stackrel{J_{1}}{\rightarrow} P$ and $S_{1} \stackrel{J_{2}}{\rightarrow} P$ be full realizations of the Poisson manifold $P$. Let $x_{i} \in S_{i}$ be such that $J_{1}\left(x_{1}\right)=J_{2}\left(x_{2}\right)$. Then there exist:

(i) Neighborhoods $\mathcal{Q}_{1}$ and $\mathcal{Q}_{2}$ of $s_{1}$ and $s_{2}$.

(ii) Splittings $\mathscr{U}_{1} \approx \delta_{1} \times \mathcal{V}_{1}$ and $\mathscr{U}_{2}=\delta_{J_{i}} \times \mathcal{V}_{2}$ such that $\left.J_{i}\right|_{u_{i}}$ depends only on the $\mathcal{S}_{i}^{\prime}$ components, via a Poisson map, $\mathcal{S}_{i} \stackrel{J_{i}}{\rightarrow} P$.

(iii) A canonical transformation $\phi: \mathfrak{S}_{1} \rightarrow \mathfrak{S}_{2}$ such that $J_{1}=J_{2} \circ \phi$.

In other words, any two full realizations of a Poisson manifold are equivalent up to canonical transformations and the addition of inessential canonical variables.

Proof. We begin by splitting off symplectic factors. For the realization $S_{1} \stackrel{J_{1}}{\rightarrow} P$, we find functions $\left(q_{1}, \cdots, q_{k}, p_{1}, \cdots, p_{k}, q_{1}^{\prime}, \cdots, q_{l}^{\prime}, p_{1}^{\prime}, \cdots, p_{l}^{\prime}, y_{1}, \cdots, y_{s}\right.$, $\left.y_{1}^{\prime}, \cdots, y_{t}^{\prime}\right)$ on a neighborhood $\mathcal{Q}_{1}$ of $x_{1}$ as in the proof of Theorem 8.1. The functions $\left(q, p, q^{\prime}, p^{\prime}\right)$ map $\mathcal{U}_{1}$ onto an open set $\mathscr{V}_{1}$ in $\mathbf{R}^{2(k+l)}$. If we take $\mathcal{S}_{1}$ to be the quotient of $\mathcal{Q}_{1}$ by the hamiltonian flows of $q, p, q^{\prime}$ and $p^{\prime}$, there is a natural isomorphism of $\mathscr{U}_{1}$ with $\delta_{1} \times \mathscr{V}_{1}$, and the functions $y_{1}, \cdots, y_{s}$ and $y_{1}^{\prime}, \cdots, y_{s}^{\prime}$ all live on $\mathcal{S}_{1}$, where they generate polar function groups. After doing the same for $S_{2} \stackrel{J_{2}}{\rightarrow} P$, we have reduced the problem to the case where $P$ has rank zero at $J_{i}\left(x_{i}\right)$, and $\operatorname{dim} S_{1}=\operatorname{dim} S_{2}=2 \operatorname{dim} P$, i.e., we are dealing with realizations of minimal dimension.

Now we may choose for each $i$ a lagrangian section $\sigma_{i}$ of $J_{i}$ though $x_{i}$, i.e., a lagrangian embedding $P \stackrel{\sigma_{i}}{\rightarrow} S_{i}$ such that $J_{i} \circ \sigma_{i}=$ identity and $\sigma_{i}\left(J_{i}\left(x_{i}\right)\right)=x_{i}$.

Next let $\left(y_{1}, \cdots, y_{s}\right)$ be coordinates on $P$, and $\left(z_{1}, \cdots, z_{s}\right)$ the dual coordinates on the dual space $P^{*}$. For each $z$, a function $f_{z, i}: S \rightarrow \mathbf{R}$ may be defined by $f_{z, i}(y)=\langle z, y\rangle \circ J_{i}$; by Lemma 2.1 its hamiltonian flow $\Psi_{z, i}^{t}$ covers the hamiltonian flow $\chi_{z}^{t}$ of $\langle z, y\rangle$ on $P$. We map $P \times P^{*}$ (locally) to $S_{i}$ by 
$\lambda_{i}:(y, z) \rightarrow \Psi_{z, i}^{t}\left(\sigma_{i}(y)\right)$, and set $\phi=\lambda_{2} \circ \lambda_{1}^{-1}: S_{1} \rightarrow S_{2}$. It follows from our construction that $J_{2} \circ \phi=J_{1}$.

To show that $\phi$ is canonical, we will show that $\lambda_{1}^{*} \Omega_{1}=\lambda_{2}^{*} \Omega_{2}$, where $\Omega_{i}$ is the symplectic structure on $S_{i}$. First we calculate the pulled back forms along the "zero section" $z=0$. There we have

$$
\left(T \lambda_{i}\right)\left(\frac{\partial}{\partial y_{j}}\right)=\left(T \sigma_{i}\right) \frac{\partial}{\partial y_{j}}, \quad\left(T \lambda_{i}\right)\left(\frac{\partial}{\partial z_{j}}\right)=\xi_{y_{j} \circ J_{i}} \circ \sigma_{i} .
$$

Thus

$$
\left(\lambda_{i}^{*} \Omega_{i}\right)\left(\frac{\partial}{\partial y_{j}}, \frac{\partial}{\partial y_{k}}\right)=\Omega_{i}\left(\left(T \sigma_{i}\right)\left(\frac{\partial}{\partial y_{j}}\right),\left(T \sigma_{i}\right)\left(\frac{\partial}{\partial y_{k}}\right)\right)=0,
$$

since $\sigma_{i}$ is lagrangian. Similarly,

$$
\begin{aligned}
\left(\lambda_{i}^{*} \Omega_{i}\right)\left(\frac{\partial}{\partial z_{j}}, \frac{\partial}{\partial z_{k}}\right) & =\Omega_{i}\left(\xi_{y_{j}} \circ J_{i}, \xi_{y_{k} \circ J_{i}}\right) \\
& =-\left\{y_{j} \circ J_{i}, y_{k} \circ J_{i}\right\} \circ \sigma_{i} \\
& =-\left\{y_{j}, y_{k}\right\} \circ J_{i} \circ \sigma_{i}=-\left\{y_{j}, y_{k}\right\} \\
\left(\lambda_{i}^{*} \Omega_{i}\right)\left(\frac{\partial}{\partial y_{j}}, \frac{\partial}{\partial z_{k}}\right)= & \Omega_{i}\left(\left(T \sigma_{i}\right)\left(\frac{\partial}{\partial y_{j}}\right), \xi_{y_{k}} \circ J_{i}\right) \\
= & -\left(T \sigma_{i}\right)\left(\frac{\partial}{\partial y_{j}}\right) \cdot y_{k} \circ J_{i}=-\frac{\partial y_{k}}{\partial y_{j}}=-\delta_{k j}
\end{aligned}
$$

We note that all these results are independent of $i$, so $\lambda_{1}^{*} \Omega_{1}=\lambda_{2}^{*} \Omega_{2}$ along the zero section. But the map $\lambda_{i}$ at all points of $P \times P^{*}$ is obtained by starting along the zero section and following trajectories of hamiltonian vector fields with respect to $\Omega_{i}$, so $\lambda_{1}^{*} \Omega_{1}=\lambda_{2}^{*} \Omega_{2}$ everywhere.

\section{The restricted three-body problem and other examples}

In this final section we shall give several illustrations of how Poisson manifolds provide a context for describing naturally arising situations in which symplectic manifolds of different topological types fit together.

A. Contractions of Lie groups. For any value of the parameter $\varepsilon$, the relations

$$
\left[X_{3}, X_{1}\right]=X_{2},\left[X_{2}, X_{3}\right]=X_{1},\left[X_{1}, X_{2}\right]=\varepsilon X_{3}
$$

describe a 3-dimensional Lie algebra. The algebra is isomorphic to $\mathfrak{g} \mathfrak{o}(3)$ for $\varepsilon>0$ and to $\mathfrak{g l}(2, \mathbf{R})$ for $\varepsilon<0$, and is the algebra $e(2)$ of the Euclidean motion 
group of the plane for $\varepsilon=0$. The corresponding bracket relations

$$
\left\{x_{3}, x_{1}\right\}=x_{2},\left\{x_{2}, x_{3}\right\}=x_{1},\left\{x_{1}, x_{2}\right\}=\varepsilon x_{3}
$$

describe a 1-parameter family of Lie-Poisson structures on $\mathbf{R}^{3}$.

There is another interpretation of (1). If we consider $\varepsilon$ as a fourth variable in addition to $\left(x_{1}, x_{2}, x_{3}\right)$, and we add the relations

$$
\left\{\varepsilon, x_{1}\right\}=\left\{\varepsilon, x_{2}\right\}=\left\{\varepsilon, x_{3}\right\}=0,
$$

then (1) and (2) together describe a Poisson structure on $\mathbf{R}^{4}$, which is not of Lie-Poisson type but includes as Poisson submanifolds all the members of the family described by (1). In particular, the symplectic leaves of (1) for various values of $\varepsilon$ (spheres for $\varepsilon>0$, cylinders for $\varepsilon=0$, and hyperboloids for $\varepsilon>0$ ) are all included as the symplectic leaves for (1) and (2). The two Casimir functions whose level manifolds are those symplectic leaves are $\varepsilon$ and $x_{1}^{2}+x_{2}^{2}$ $+\varepsilon x_{3}^{2}$.

It is interesting to look at the linearized structures at points where the rank is zero. (Points of rank two are all regular.) Denoting by $\left(y_{1}, y_{2}, y_{3}, \delta\right)$ the coordinates on the tangent space, we may consider two cases. In each case, $\delta$ is a Casimir function.

(i) $x_{1}=x_{2}=x_{3}=0$. We have

$$
\left\{y_{3}, y_{1}\right\}=y_{2},\left\{y_{2}, y_{3}\right\}=y_{1},\left\{y_{1}, y_{2}\right\}=\varepsilon y_{3} \text {. }
$$

Since $\varepsilon$ is again a parameter here, the Lie algebra is the product of $\mathbf{R}$ with $\mathfrak{s o}(3), \mathfrak{s} \mathfrak{l}(2, \mathbf{R})$, or $\mathrm{e}(2)$.

(ii) $x_{1}=x_{2}=\varepsilon=0, x_{3} \neq 0$. We have

$$
\left\{y_{3}, y_{1}\right\}=y_{2},\left\{y_{2}, y_{3}\right\}=y_{1},\left\{y_{1}, y_{2}\right\}=x_{3} \delta \text {. }
$$

This time the algebra is the four-dimensional "oscillator algebra" with the parameter $x_{3}$ playing the role of Planck's constant.

Similar constructions and analysis can be carried out whenever one has a family of Lie algebra structures depending upon one or more parameters, such as the degeneration or contraction of the Poincaré group to the Galilean group as the parameter (speed of light) ${ }^{-1}$ approaches zero.

B. The light top. The motion of a rigid body, anchored at one point, in a gravitational field is given by a hamiltonian system on the Lie-Poisson manifold $e(3)^{*}$, where $E(3)$ is the Euclidean motion group in $\mathbf{R}^{3}$. The bracket relations on $e(3)^{*}$ are

$$
\begin{array}{lll}
\left\{M_{2}, M_{3}\right\}=M_{1}, & \left\{M_{3}, M_{1}\right\}=M_{2}, & \left\{M_{1}, M_{2}\right\}=M_{3}, \\
\left\{M_{2}, F_{3}\right\}=F_{1}, \quad\left\{M_{3}, F_{1}\right\}=F_{2}, & \left\{M_{1}, F_{2}\right\}=F_{3}, \\
\left\{F_{2}, M_{3}\right\}=F_{1}, \quad\left\{F_{3}, M_{1}\right\}=F_{2}, & \left\{F_{1}, M_{2}\right\}=F_{3}, \\
\left\{F_{i}, F_{j}\right\}=\left\{F_{i}, M_{i}\right\}=0 .
\end{array}
$$


The vector $\mathbf{M}=\left(M_{1}, M_{2}, M_{3}\right)$ is the angular momentum in body coordinates; $\mathbf{F}=\left(F_{1}, F_{2}, F_{3}\right)$ is the gravitational acceleration vector times the mass of the body, again in body coordinates.

The hamiltonian for the Euler-Poisson equations of rigid body motion is $H=\frac{1}{2} \sum M_{i}^{2} / I_{i}+\sum x_{i} F_{i}$, where $I_{1}, I_{2}, I_{3}$ are the principal moments of inertia, and $\left(x_{1}, x_{2}, x_{3}\right)$ is the vector to the body's center of mass from the anchor point. (See Guillemin and Sternberg [15] or Holmes and Marsden [21].)

The Casimir functions on $\mathrm{e}(3)^{*}$ are generated by $\|\mathbf{F}\|^{2}=F_{1}+F_{2}+F_{3}$ and $\mathbf{F} \cdot \mathbf{M}=F_{1} M_{1}+F_{2} M_{2}+F_{3} M_{3}$. The regular symplectic leaves (coadjoint orbits), given by specifying a nonzero value for $\|\mathbf{F}\|^{2}$ and any value for $\mathbf{F} \cdot \mathbf{M}$, are diffeomorphic to $T^{*} S^{2}$ but with varying symplectic structures. The singular symplectic leaves lie in the 3-dimensional Poisson submanifold $\mathbf{F}=0$ and are just the $S O(3)$ coadjoint orbits $\|\mathbf{M}\|^{2}=$ constant. Physically, these leaves represent the situation where the force is turned off (or the gravitational mass of the body is zero.) As explained in Arnold [3], this limit is equivalent to the limit of infinitely fast rotation.

If we linearize at the leaf $\mathbf{F}=0,\|\mathbf{M}\|=M$, we get a transverse Poisson structure equal to the Lie-Poisson structure for the coadjoint isotropy group of $\left(\mathbf{O}, \mathbf{M}_{0}\right)$. This isotropy group turns out to consist of rotations about the $M$-axis and all translations, so it is isomorphic to $E(2) \times \mathbf{R}$.

For simplicity we shall consider the case where $\left(0, \mathbf{M}_{0}\right)$ is a stationary point, i.e., a critical point of $H$ restricted to the symplectic leaf. Without loss of generality we may assume that $\mathbf{M}_{0}=(M, 0,0)$.

As coordinates on the transverse Lie-Poisson manifold we have $\left\{N_{1}, G_{1}, G_{2}, G_{3}\right\}$ with relations

$$
\left\{N_{1}, G_{2}\right\}=G_{3},\left\{N_{1}, G_{3}\right\}=-G_{2},
$$

and all other brackets zero. The linearized hamiltonian is $H^{\prime}=M N_{1} / I_{1}+$ $x_{2} G_{2}+x_{3} G_{3}$ yielding the equations of motion

$$
\dot{G}_{1}=0, \dot{G}_{2}=-M G_{3} / I_{1}, \dot{G}_{3}=M G_{2} / I_{1}, \dot{N}_{1}=x_{2} G_{3}-x_{3} G_{2} .
$$

Each motion is confined to an $E(2) \times \mathbf{R}$ coadjoint orbit, which is a cylinder $G_{1}=$ constant, $G_{2}^{2}+G_{3}^{2}=$ constant, and it lies in a plane $H^{\prime}=$ constant, so the trajectories are circles, around which the phase point moves with the precession-nutation frequency $\omega=M / I_{1}$. Whether the center of mass is on the rotation axis or not $\left(x_{2}^{2}+x_{3}^{2}=\right.$ or $\left.\neq 0\right)$ determines whether $N_{1}$ oscillates with $\left(G_{2}, G_{3}\right)$, but the motion is qualitatively the same in either case.

C. The restricted three-body problem. The phase space for the gravitational $n$-body problem is the standard symplectic space $\mathbf{R}^{6 N}$ with coordinates 
$\left(\mathbf{q}_{1}, \cdots, \mathbf{q}_{N}, \mathbf{p}_{1}, \cdots, \mathbf{p}_{N}\right)$, each $\mathbf{q}_{i}$ and $\mathbf{p}_{i}$ being a vector in $\mathbf{R}^{3}$. The hamiltonian is

$$
H=\frac{1}{2} \sum_{i} \frac{p_{i}^{2}}{m_{i}}-\sum_{i<j} m_{i} m_{j}\left\|\mathbf{q}_{i}-\mathbf{q}_{j}\right\|^{-1} .
$$

The restricted three-body problem is the "limit" in the case $N=3$, as the mass $m_{3}$ goes to zero. The first two ("primary") bodies move as in a two-body problem, while the third body moves in the time-varying gravitational field of the first two. If the two primary bodies are in circular orbits, the motion of the third body is a time-independent hamiltonian system when viewed in a coordinate system which rotates with the primaries; the hamiltonian for this motion is called the Jacobi integral (see Abraham and Marsden [1]).

Our aim here is to show how the symplectic structure for the restricted three-body problem is related to that of the unrestricted problem. Curiously it is necessary for us to do this via an infinite-dimensional system called the Poisson-Vlasov equation. ${ }^{13}$ This happens because no finite-dimensional Lie group of canonical transformations is triply transitive on $\mathbf{R}^{\mathbf{6}}$.

The basic phase space for the Poisson-Vlasov equation (Gibbons [10], Marsden and Weinstein [32], Morrison [35]) is the Lie-Poisson algebra $\mathscr{D}^{\prime}\left(\mathbf{R}^{6}\right)$ of densities $f(q, v) d q d v$ on $\mathbf{R}^{6}$, dual to the Lie algebra $C^{\infty}\left(\mathbf{R}^{6}\right)$ of functions under the canonical Poisson bracket. ${ }^{14}$ Denoting the bracket on $\mathscr{D}^{\prime}\left(\mathbf{R}^{6}\right)$ by $\{\{\}$,$\} to distinguish it from the canonical bracket \{$,$\} , we have$

$$
\{\{F, G\}\}(f)=\int_{\mathbf{R}^{6}} f\left\{\frac{\delta F}{\delta f}, \frac{\delta G}{\delta f}\right\} d q d v,
$$

where $\delta F / \delta f$ is the "functional derivative" defined by

$$
\lim _{\varepsilon \rightarrow 0} \frac{1}{\varepsilon}[F(f+\varepsilon g)-F(f)]=\int_{\mathbf{R}^{6}} g(x, v) \frac{\delta F}{\delta f}(x, v) d x d v .
$$

The corresponding Lie group is the group $\operatorname{Can}\left(\mathbf{R}^{6}\right)$ of canonical transformations on $\mathbf{R}^{6}$, and the symplectic leaves in $\mathscr{D}^{\prime}\left(\mathbf{R}^{6}\right)$ are the classes of densities which are equivalent under canonical transformations. ${ }^{15}$

The typical symplectic leaf is infinite dimensional, but there are also $6 N$-dimensional leaves $S_{\left(m_{1}, \cdots, m_{N}\right)}$ consisting of delta densities of the form

\footnotetext{
${ }^{13}$ The equation is also called the collisionless Boltzmann equation, or the equation of stellar dynamics. For the electrostatic (repulsive) problem, the sign of the potential in $H$ should be reversed.

${ }^{14}$ Our "fundamental" particles will be taken to have unit mass. "Bodies" will be (not necessarily integer) multiplies of these.

${ }^{15}$ Actually a group whose Lie algebra is $C^{\infty}\left(\mathbf{R}^{6}\right)$ must be a one-dimensional central extension of Can $\left(\mathbf{R}^{6}\right)$, but the coadjoint representation factors through $\operatorname{Can}\left(\mathbf{R}^{6}\right)$ anyway.
} 
$\sum_{i=1}^{N} m_{i} \delta\left(\mathbf{q}-\mathbf{q}_{i}, \mathbf{v}-\mathbf{v}_{i}\right)$, where $\left(\mathbf{q}_{1}, \mathbf{v}_{1}\right), \cdots,\left(\mathbf{q}_{N}, \mathbf{v}_{N}\right)$ are $N$ distinct points in $\mathbf{R}^{6}$ and $m_{1}, \cdots, m_{N}$ are given positive real numbers. Then $\operatorname{Can} \mathbf{R}^{6}$ acts on the product $\mathbf{R}^{6 N}$ with symplectic structure $\sum_{i=1}^{N} m_{i} d \mathbf{q}_{i} \wedge d \mathbf{v}_{i}$; the momentum mapping of this action, restricted to the set $\tilde{S}_{\left(m_{1}, \cdots, m_{N}\right)}$ of non-repeating $N$-tuples in $\mathbf{R}^{6 N}$ is an $N$ !-fold covering map onto $S_{\left(m_{1}, \cdots, m_{N}\right)}$. Writing $\mathbf{p}_{i}$ for $m_{i} \mathbf{v}_{i}$, we find that $\tilde{S}_{\left(m_{1}, \cdots, m_{N}\right)}$ may be identified with the phase space of the $N$-body problem.

The hamiltonian for the Poisson-Vlasov equation is the functional

$$
\begin{aligned}
H(f) & =\frac{1}{2} \int_{\mathbf{R}^{6}}\|\mathbf{v}\|^{2} f(q, \mathbf{v}) d q d v \\
& =\int_{\mathbf{R}^{6} \times \mathbf{R}^{6}}\left\|q_{1}-q_{2}\right\|^{-1} f\left(\mathbf{q}_{1}, \mathbf{v}_{1}\right) f\left(\mathbf{q}_{2}, \mathbf{v}_{2}\right) d \mathbf{q}_{2} d \mathbf{v}_{2} .
\end{aligned}
$$

The hamiltonian flow for $H$ is given by

$$
\dot{f}=\left\{f, \frac{\delta H}{\delta f}\right\}=\left\{f, \frac{1}{2}\|\mathbf{v}\|^{2}+V_{f}\right\},
$$

where

$$
V_{f}(\mathbf{q})=-\int_{\mathbf{R}^{6}}\left\|\mathbf{q}_{1}-\mathbf{q}\right\|^{-1} f\left(\mathbf{q}_{1}, \mathbf{v}_{1}\right) d q_{1} d v_{1} .
$$

(2) is a Liouville equation for the evolution of the density $f$ in phase space under the flow of the $f$-dependent hamiltonian $\frac{1}{2}\|\mathbf{v}\|^{2}+V_{f}$. In particular, the delta densities on the orbits $S_{m_{1}, \cdots, m_{N}}$ evolve under the flow of the $N$-body problem with masses $m_{1}, \cdots, m_{N}$. Thus we have embedded all the $N$-body problems in a single equation-the Poisson-Vlasov equation.

Although the flow (2) restricts without difficulty to the orbits $S_{m_{1}, \cdots, m_{N}}$, things are more complicated when we try to restrict the hamiltonian (1). Formally, we obtain

$$
H\left(\sum_{i=1}^{N} m_{i} \delta\left(\mathbf{q}-\mathbf{q}_{i}, \mathbf{v}-\mathbf{v}_{i}\right)\right)=\frac{1}{2} \sum_{i=1}^{N} m_{i}\left\|\mathbf{v}_{i}\right\|^{2}+\frac{1}{2} \sum_{i, j=1}^{N} m_{i} m_{j}\left\|\mathbf{q}_{i}-\mathbf{q}_{j}\right\|^{-1} .
$$

The problem with (3) lies in the infinite "self-energy" terms with $i=j$ in the second sum. If we "renormalize" the hamiltonian by simply deleting these terms, the result is the usual hamiltonian for the $N$-body problem. It is not clear how best to justify this renormalization, but the essential idea should be that a hamiltonian flow on $\mathscr{D}^{\prime}\left(\mathbf{R}^{6}\right)$ is unaltered if we subtract a Casimir function from the hamiltonian. The Casimir function (a typical one is $\left.\int_{\mathbf{R}^{6}}[f(\mathbf{x}, \mathbf{v})]^{p} d x d v\right)$ should be selected so as to approximate the large self-energy terms as the delta densities are approximated by smooth bump functions. 
Putting aside the renormalization question, we are ready to take some limits. If we let the last mass $m_{N}$ approach zero, then the $6 N$-dimensional leaves $S_{m_{1}, \cdots, m_{N}}$ close in on the $6(N-1)$-dimensional leaf $S_{m_{1}, \cdots, m_{N}-1}$. Thus, in particular, the phase space of the two-body problem is exhibited as a limit of phase spaces of three-body problems.

To obtain the restricted three-body problem, we must linearize about $S_{m_{1}, m_{2}}$. According to the theory in $\$ 4$, the transverse Poisson structure at the point $\sum_{i=1}^{2} m_{i} \delta\left(\mathbf{q}-\mathbf{q}_{i}, \mathbf{v}-\mathbf{v}_{i}\right)$ is the Lie-Poisson manifold $\mathrm{g}^{*}$, where $\mathrm{g} \subseteq C^{\infty}\left(\mathbf{R}^{6}\right)$ is the subalgebra consisting of those functions having a critical point at $\left(\mathbf{q}_{1}, \mathbf{v}_{1}\right)$ and $\left(\mathbf{q}_{2}, \mathbf{v}_{2}\right)$. Thus $g^{*}$ consists of densities on $\mathbf{R}^{6}$ modulo some loss of information at $\left(\mathbf{q}_{1}, \mathbf{v}_{1}\right)$ and $\left(\mathbf{q}_{2}, \mathbf{v}_{2}\right)$. In particular, $g^{*}$ contains a coadjoint orbit of the form $S_{m}=\left\{m \delta\left(\mathbf{q}-\mathbf{q}_{3}, \mathbf{v}-\mathbf{v}_{3}\right) \mid\left(\mathbf{q}_{3}, \mathbf{v}_{3}\right)\right.$ is different from $\left(\mathbf{q}_{1}, \mathbf{v}_{1}\right)$ and $\left.\left(\mathbf{q}_{2}, \mathbf{v}_{2}\right)\right\}$. $S_{m}$ is just the phase space for the restricted three-body problem in which the third body has "infinitesimal mass $m$." Its symplectic structure is $m d \mathbf{q} \wedge d \mathbf{v}$.

Now we apply the theory of $\S 5$. If we linearize along an arbitrary trajectory of the two-body problem, the result is a time-dependent hamiltonian system in $S_{m}$. If the motion of the two bodies is circular, though, it can be identified with a trajectory of a symmetry group generated by the functional

$$
K(f)=\int_{\mathbf{R}^{6}} f(\mathbf{x}, \mathbf{v})\left[\left(\left(\mathbf{x}-\mathbf{x}_{0}\right) \times \mathbf{v}\right) \cdot \omega\right] d x d v,
$$

where $\mathbf{x}_{0}$ is the center of mass of the two-body system, and $\omega$ is its angular velocity vector. Subtracting $K(f)$ from $H(f)$ (renormalized), linearizing in the transverse space, and restricting to the coadjoint orbit $S_{m}$, we obtain precisely the Jacobi integral. Thus we have recovered the full hamiltonian structure of the circular restricted three-body problem.

\section{References}

[1] R. Abraham \& J. Marsden, Foundations of mechanics, 2nd edition, Benjamin Cummings, Reading, Massaschusetts, 1978.

[2] R. Abraham \& J. Robbin, Transversal mappings and flows, Benjamin, New York, 1967.

[3] V. I. Arnold, Mathematical methods of classical mechanics, Graduate Text in Math., Vol. 60, Springer, New York, 1978.

[4] F. A. Berezin, Some remarks about the associated envelope of a Lie algebra, Funct. Anal. Appl. 1 (1967) 91-102.

[5] — Quantization, Izv. Akad. Nauk. SSSR, Ser. Matem. 38 (1974) 1116-1175 (= Math. USSR Izv. 38 (1974) 1109-1164).

[6] R. Berger, Géométrie algébrique de Poisson et déformations, Publ. Dept. Math. Univ. Claude Bernard, Lyon 16, fasc. 2, 1979, 1-69.

[7] R. L. Bishop \& R. J. Crittenden, Geometry of manifolds, Academic Press, New York, 1964.

[8] M. Duflo \& M. Vergne, Une propriété de la représentation coadjointe d'une algebre de Lie, $\mathrm{C}$. R. Acad. Sci. Paris, Sér. A-B, 268 (1969) A583-A585.

[9] H. Freudenthal \& H. deVries, Linear Lie groups, Academic Press, New York, 1969. 
[10] J. Gibbons, Collisionless Boltzmann equations and integrable moment equations, Physica 3D (1981) 503-511.

[11] M. Golubitsky \& V. Guillemin, Stable mappings and their singularities, Graduate Texts in Math., Vol. 14, Springer, New York, 1973.

[12] M. Gotay, R. Lashof, J. Sniatycki \& A. Weinstein, Closed forms on symplectic fibre bundles, Comment. Math. Helv., to appear.

[13] V. W. Guillemin, Band asymptotics in two dimensions, Advances in Math. 42 (1981) 248-282.

[14] V. Guillemin \& S. Sternberg, Remarks on a paper of Hermann, Trans. Amer. Math. Soc. 130 (1968) 110-116.

[15] _ The moment map and collective motion, Ann. Physics 127 (1980) 220-253.

[16] S. Helgason, Differential geometry and symmetric spaces, Academic Press, New York, 1962.

[17] R. Hermann, Cartan connections and the equivalence problem for geometric structures, Contributions to Differential Equations 3 (1964) 199-248.

[18] The formal linearization of a semisimple Lie algebra of vector fields about a singular point, Trans. Amer. Math. Soc. 130 (1968) 105-109.

[19] - Gauge fields and Cartan-Ehresmann connections. Part A: Interdisciplinary mathematics, Vol. X, Math. Sci. Press, Brookline, 1975.

[20] _ Toda lattices, cosymplectic manifolds, Bäcklund transformations and kinks. Part A: Interdisciplinary mathematics, Vol. XV, Math. Sci. Press, Brookline, 1977.

[21] P. J. Holmes \& J. E. Marsden, Horseshoes and Arnold diffusion for Hamiltonian systems on Lie groups, Indiana Univ. Math. 32 (1983) 273-309.

[22] N. Jacobson, Lie algebras, Interscience, New York, 1962.

[23] D. Kazhdan, B. Kostant \& S. Sternberg, Hamiltonian group actions and dynamical systems of Calogero type, Comm. Pure Appl. Math. 31 (1978) 481-508.

[24] A. A. Kirillov, Unitary representations of nilpotent Lie groups, Russian Math. Surveys 17 (1962) 53-104 (= Usp. Mat. Nauk. 17 (1962) 57-110).

[25] _ Local Lie algebras, Russian Math. Surveys 31 (1976) 56-75 (= Uspekhi Mat. Nauk. 31 (1976) 57-76).

[26] B. Kostant, Orbits, symplectic structures, and representation theory, Proc. U.S.-Japan Seminar in Differential Geometry, Kyoto, Japan, 1965, Nippon Hyoronisha Tokyo, 1965, 71.

[27] _ Quantization and unitary representations. Part I: Prequantization, Lecture Notes in Math. Vol. 170, Springer, Berlin, 1970, 87-208.

[28] A. Lichnerowicz, Les variétiés de Poisson et leurs algèbres de Lie associées, J. Differential Geometry 12 (1977) 253-300.

[29] S. Lie, Theorie der transformationsgruppen, (Zweiter Abschnitt, unter mitwirkung von Prof. Dr. Friedrich Engel), Teubner, Leipzig, 1890.

[30] B. Malgrange, Equation de Lie. II, J. Differential Geometry 7 (1972) 117-141.

[31] J. Marsden \& A. Weinstein, Reduction of symplectic manifolds with symmetry, Rep. Mathematical Phys. 5 (1974) 121-130.

[32] _ Coadjoint orbits, vortices, and Clebsch variables for incompressible fluids, Proc. of the Conference on Order in Chaos, Los Alamos, 1982, A. Scott, ed., Physica, to appear.

[33] J. Marsden, T. Ratiu \& A. Weinstein, Semi-direct products and reduction in mechanics, to appear in Trans. Amer. Math. Soc.

[34] A. S. Mishchenko \& A. T. Fomenko, Generalized Liouville method of integration of Hamiltonian systems, Funct. Anal. Appl. 12 (1978) 113-121 (= Funkt. Anal. Prilozh. 12 (1978) 46-56).

[35] P. J. Morrison, The Maxwell-Vlasov equations as a continuous Hamiltonian system, Phys. Lett. 80A (1980) 383-386.

[36] J. Moser, On the volume elements on a manifold, Trans. Amer. Math. Soc. 120 (1965) 286-294. 
[37] R. Moussu, Sur l'existence d'intégrales premières pour un germe de forme de Pfaff, Ann. Inst. Fourier (Grenoble) 26 (1976) 171-120.

[38] G. Reeb, Sur certaines propriétés topologiques des variétés feuilletées, Hermann, Paris, 1952.

[39] J. Roels \& A. Weinstein, On functions whose Poisson brackets are constant, J. Mathematical Phys. 12 (1971) 1482-1486.

[40] W. Schmid, Poincaré and Lie groups, Bull. Amer. Math. Soc. 6 (1982) 175-186.

[41] J. M. Souriau, Quantification géometrique, Comm. Math. Phys. 1 (1966) 374-398.

[42] J. A. Schouten, Über differentialkomitanten zweier kontravarianter Grössen, Nederl. Akad. Wetensch. Proc. Ser. A, 43 (1940) 449-452.

[43] M. Tabor \& Y. M. Treve (eds.), Mathematical methods in hydro-dynamics and integrability in dynamical systems, Proc. Amer. Inst. Phys. Conference 88, 1982.

[44] W. M. Tulczyjew, Poisson brackets and canonical manifolds, Bull. Acad. Polon. Sci. Sér. Sci. Math. Astronom. 22 (1974) 931-934.

[45] J. Vey, Sur le lemme de Morse, Invent. Math. 40 (1977) 1-10.

[46] A. M. Vinogradov \& I. S. Krasilshchik, What is the Hamiltonian formalism? Russian Math. Surveys 30 (1975) 117-202 (= Uspehkhi Mat. Nauk. 30 (1975) 173-198).

[47] A. Weinstein, Symplectic manifolds and their lagrangian submanifolds, Advances in Math. 6 (1971) 329-346.

[48] __ Fat bundles and symplectic manifolds, Advances in Math. 37 (1971) 239-250.

UNIVERSITY OF CALIFORNIA, BERKELEY 
